\title{
Chebyshev Wavelet Finite Difference Method: A New Approach for Solving Initial and Boundary Value Problems of Fractional Order
}

\author{
A. Kazemi Nasab, ${ }^{1}$ A. Kılıçman, ${ }^{1}$ Z. Pashazadeh Atabakan, ${ }^{1}$ and S. Abbasbandy ${ }^{2}$ \\ ${ }^{1}$ Department of Mathematics, University Putra Malaysia (UPM), 43400 Serdang, Selangor, Malaysia \\ ${ }^{2}$ Department of Mathematics, Imam Khomeini International University, Ghazvin 34149, Iran
}

Correspondence should be addressed to A. K1lıçman; kilicman@yahoo.com

Received 25 April 2013; Revised 30 August 2013; Accepted 9 September 2013

Academic Editor: Andrew Pickering

Copyright (C) 2013 A. Kazemi Nasab et al. This is an open access article distributed under the Creative Commons Attribution License, which permits unrestricted use, distribution, and reproduction in any medium, provided the original work is properly cited.

\begin{abstract}
A new method based on a hybrid of Chebyshev wavelets and finite difference methods is introduced for solving linear and nonlinear fractional differential equations. The useful properties of the Chebyshev wavelets and finite difference method are utilized to reduce the computation of the problem to a set of linear or nonlinear algebraic equations. This method can be considered as a nonuniform finite difference method. Some examples are given to verify and illustrate the efficiency and simplicity of the proposed method.
\end{abstract}

\section{Introduction}

The study of fractional calculus dates back to 17th century, starting by G. W. Leibnitz $(1695,1697)$ and L. Euler (1730) $[1,2]$ and then has been developed by many researchers in different disciplines. In the year 1823, Liouville and Abel introduced the theory of fractional derivatives and integrals; for more details, please refer to $[3,4]$. Fractional calculus has received much more attention from scientists and engineers in recent years. Many researchers in various fields found that derivatives of noninteger order are useful for the description of some natural physics phenomena and dynamic system processes such as damping laws and diffusion process $[5,6]$. In general, it is difficult to solve fractional differential equations analytically. Therefore, it is necessary to introduce some reliable and efficient numerical algorithms to solve them. During the past decades, an increasing number of numerical methods were being developed. These methods include homotopy analysis method [7], homotopy perturbation method [810], variational iteration method [9-13], finite difference method [5, 14-18], Adomian decomposition method [19-23], fractional differential transform method [24, 25], predictorcorrector method [26], fractional linear multistep method [27], extrapolation method [28], integral transform [29], and generalized block pulse operational matrix method [30,31].
In recent years, wavelets have received considerable attention by researchers in different fields of science and engineering. One advantage of wavelet analysis is the ability to perform local analysis [32]. Wavelet analysis is able to reveal signal aspects that other analysis methods miss, such as trends, breakdown points, and discontinuities. In comparison with other orthogonal functions, multiresolution analysis aspect of wavelets permits the accurate representation of a variety of functions and operators. In other words, we can change $M$ and $k$ simultaneously to get more accurate solution. Another benefit of wavelet method for solving equations is that after discreting the coefficients matrix of algebraic equations is sparse. So the use of wavelet methods for solving equations is computationally efficient. In addition, the solution is convergent. The operational matrix of fractional order integration for the Chebyshev wavelet, Legendre wavelet, and Haar wavelet has been introduced in [33-35] to solve the differential equations of fractional order. A CAS wavelet operational matrix of fractional order integration has been developed by Saeedi et al., to solve fractional nonlinear integrodifferential equations [36,37].

The paper is organized as follows. Section 2 included some necessary definitions and mathematical preliminaries of fractional calculus, Chebyshev polynomials, and Chebyshev wavelets. In Section 3, we introduce the Chebyshev finite 
difference method. In Section 4, Chebyshev wavelet finite difference method (CWFD) is presented. Section 5 included convergence analysis of the proposed method. In Section 6, the proposed approach is used to approximate the fractional differential equations. As a result the fractional differential equation is converted to a system of algebraic equations which is simply solved. Some illustrative examples of different types are given to demonstrate the efficiency and accuracy of the method in Section 7. In Section 8, concluding remarks are given.

\section{Preliminaries and Notations}

In this section, we present some notations, definitions, and preliminary facts that will be used further in this work.

2.1. Fractional Integral and Derivative. There are several definitions of fractional integral and derivatives [2, 38, 39], including Riemann-Liouville, Caputo, Weyl, Hadamard, Marchaud, Riesz, Grunwald-Letnikov, and Erdelyi-Kober. The most commonly used definition is of Riemann-Liouville and Caputo. One of the drawbacks of Riemann-Liouville method is that it cannot incorporate the nonzero initial condition at lower limit. The Caputo fractional derivative allows the utilization of initial and boundary conditions involving integer order derivatives, which have clear physical interpretations. Therefore, in this study we will use the Caputo fractional derivative $D^{\alpha}$ proposed by Podlubny [14].

Definition 1. A real function $f(x), x>0$, is said to be in the space $C_{\mu}, \mu \in \mathbb{R}$ if there exists a real number $p>\mu$ such that $f(x)=x^{p} f_{1}(x)$, where $f_{1}(x) \in C[0, \infty)$, and it is said to be in the space $C_{\mu}^{n}$ as if and only if $f^{n}(x) \in C_{\mu}, n \in \mathbb{N}$.

Definition 2. The Riemann-Liouville fractional integration of order $\alpha \geq 0$ of a function $f \in C_{\mu}, \mu \geq-1$ is defined as [14]

$$
\begin{aligned}
& \left(I^{\alpha} f\right)(x)=\frac{1}{\Gamma(\alpha)} \int_{0}^{x}(x-\tau)^{\alpha-1} f(\tau) d \tau \\
& \left(I^{0} f\right)(x)=f(x) .
\end{aligned}
$$

Definition 3. The fractional derivative of $f(x)$ in the Caputo sense is defined as [14]

$$
\begin{aligned}
\left(D^{\alpha} f\right)(x) & =\left(I^{n-\alpha} f^{(n)}\right)(x) \\
& =\frac{1}{\Gamma(n-\alpha)} \int_{0}^{x}(x-\tau)^{n-\alpha-1} f^{(n)}(\tau) d \tau,
\end{aligned}
$$

for $n-1<\alpha \leq n, n \in \mathbb{N}, x>0, f \in C_{-1}^{n}$.

Some basic properties of the fractional operator are as follows $[14,40]$, for $f \in C_{\mu}^{n}, \mu \geq-1, \alpha, \beta \geq 0$, and $\gamma \geq-1$ we have

(1) $I^{\alpha} I^{\beta} f(x)=I^{\alpha+\beta} f(x)$,

(2) $I^{\alpha} I^{\beta} f(x)=I^{\beta} I^{\alpha} f(x)$,

(3) $I^{\alpha} x^{\gamma}=(\Gamma(\gamma+1) / \Gamma(\gamma+\alpha+1)) x^{\gamma+\alpha}$,
(4) $D^{\alpha} I^{\alpha} f(x)=f(x)$,

(5) $I^{\alpha} D^{\alpha} f(x)=f(x)-\sum_{k=0}^{n-1} f^{(k)}\left(0^{+}\right)\left(x^{k} / k !\right), x>0, n-$ $1<\alpha \leq n$,

(6)

$$
D^{\alpha} x^{k}= \begin{cases}0, & k \leq\lceil\alpha\rceil, k \in \mathbb{Z}^{+} \\ \frac{\Gamma(k+1)}{\Gamma(k+1-\alpha)} x^{k-\alpha}, & k \geq\lceil\alpha\rceil, k \in \mathbb{Z}^{+},\end{cases}
$$

where $\lceil\alpha\rceil$ denotes the smallest integer greater than or equal to $\alpha$.

2.2. Chebyshev Polynomials. Chebyshev polynomials of the first kind of degree $m$ can be defined as follows:

$$
T_{m}(x)=\cos m \alpha, \quad \alpha=\arccos x,
$$

which are orthogonal with respect to the weight function $w(x)=1 / \sqrt{1-x^{2}}[41]$,

$$
\int_{-1}^{1} T_{m}(x) T_{n}(x) w(x) d x=\frac{\pi}{2} c_{m} \delta_{n m}
$$

where $\delta_{n m}$ is the Kronecker delta function and

$$
c_{m}= \begin{cases}2, & m=0 \\ 1, & m \geq 1 .\end{cases}
$$

Chebyshev polynomials also satisfy the following recursive formula:

$$
\begin{aligned}
& T_{0}(x)=1, \quad T_{1}(x)=x, \\
& T_{m+1}(x)=2 x T_{m}(x)-T_{m-1}(x), \quad m=1,2, \ldots
\end{aligned}
$$

The set of Chebyshev polynomials is a complete orthogonal set in the Hilbert space $\mathfrak{E}_{w}^{2}[-1,1]$. A function $f \in$ $\mathfrak{E}_{w}^{2}[-1,1]$ can be written in terms of Chebyshev polynomials as

$$
\begin{aligned}
& f(x)=\sum_{m=0}^{\infty} \widehat{f}_{m} T_{m}(x), \\
& \widehat{f}_{m}=\frac{2}{\pi c_{m}} \int_{-1}^{1} f(x) T_{m}(x) w(x) d x .
\end{aligned}
$$

2.3. Wavelets and Chebyshev Wavelets. Wavelets have been very successfully used in many scientific and engineering fields. They constitute a family of functions constructed from dilation and transformation of a single function called the mother wavelet $\psi(x)$; we have the following family of continuous wavelets as $[42,43]$ :

$$
\psi_{a, b}(x)=|a|^{-1 / 2} \psi\left(\frac{x-b}{a}\right), \quad a, b \in R, a \neq 0 .
$$

If we set $a=a_{0}^{-k}, b=n b_{0} a_{0}^{-k} ; a_{0}>1, b_{0}>0$, we will get the following family of discrete wavelet which forms a wavelet basis for $L^{2}(\mathbb{R})$ :

$$
\psi_{a, b}(x)=\left|a_{0}^{k / 2}\right| \psi\left(a_{0}^{k} x-n b_{0}\right), \quad a, b \in \mathbb{Z} .
$$


In particular, when $a_{0}=2$ and $b_{0}=1$, then $\psi_{a, b}(x)$ forms an orthonormal basis.

Chebyshev wavelets $\psi_{n, m}=\psi(k, n, m, t)$ have four arguments; $n=1, \ldots, 2^{k-1}, k$ can assume any positive integer, $m$ is degree of Chebyshev polynomials of the first kind, and $t$ denotes the time. Consider

$$
\begin{aligned}
& \psi_{n, m}(x) \\
& \quad= \begin{cases}2^{k / 2} p_{m} T_{m}\left(2^{k} x-2 n+1\right), & \frac{n-1}{2^{k-1}} \leq x<\frac{n}{2^{k-1}} \\
0, & \text { otherwise, }\end{cases}
\end{aligned}
$$

where

$$
p_{m}= \begin{cases}\frac{1}{\sqrt{\pi}}, & m=0, \\ \sqrt{\frac{2}{\pi}}, & m \geq 1,\end{cases}
$$

and $m=0,1, \ldots, M$ and $n=1, \ldots, 2^{k-1}$. In (11) the coefficients are used for orthonormality. We should note that in dealing with the Chebyshev wavelets, the weight function $\widetilde{w}(x)=w(2 x-1)$ has to be dilated and translated to get orthogonal wavelets as follows:

$$
w_{n}(x)=w\left(2^{k} x-2 n+1\right) .
$$

In view of (5), Chebyshev wavelets are an orthonormal set with respect to the weight function $w_{n}(x)$ because

$$
\left\langle\psi_{n, m}(x), \psi_{r, s}(x)\right\rangle_{w_{n}}=\frac{\pi}{2} p_{m} p_{s} c_{m} \delta_{n r} \delta_{m s} .
$$

Lemma 4. The family of Chebyshev wavelets $\left\{\psi_{n, m}(x) \mid n=\right.$ $\left.1,2, \ldots, 2^{k-1}, m \in \mathbb{N} \cup\{0\}\right\}$ forms an orthonormal basis for $L^{2}[0,1]$ with respect to the weight function $w_{n}(x)$ [44].

For $k=2$ and $M=3$, Chebyshev wavelets are as follows:

$$
\left.\begin{array}{rl}
\psi_{1,0} & =\frac{2}{\sqrt{\pi}} \\
\psi_{1,1} & =\frac{2 \sqrt{2}}{\sqrt{\pi}}(4 x-1) \\
\psi_{1,2} & =\frac{2 \sqrt{2}}{\sqrt{\pi}}\left[2(4 x-1)^{2}-1\right] \\
\psi_{1,3} & =\frac{2 \sqrt{2}}{\sqrt{\pi}}\left(256 x^{3}-192 x^{2}+36 x-1\right)
\end{array}\right\} \quad 0 \leq x<\frac{1}{2}
$$

2.4. Function Approximation. A function $f(t)$ defined over $[0,1)$ may be expanded as

$$
f(x)=\sum_{n=1}^{\infty} \sum_{m=0}^{\infty} c_{n, m} \psi_{n, m}(x),
$$

where $c_{n, m}=\left(f(x), \psi_{n, m}(x)\right)_{w_{n}}$, in which $(\cdot, \cdot)$ denotes the inner product in $L_{w_{n}}^{2}[0,1]$. If we consider truncated series in (16), we obtain

$$
f(x)=\sum_{n=1}^{2^{k-1}} \sum_{m=0}^{M} c_{n, m} \psi_{n, m}(x)=C^{T} \Psi(x)
$$

where $C$ and $\Psi(x)$ are $2^{k-1}(M+1) \times 1$ matrices given by

$$
\begin{gathered}
C=\left[c_{1,0}, c_{1,1}, \ldots, c_{1, M}, c_{2,0}, c_{2,1}, \ldots, c_{2, M}, \ldots,\right. \\
\left.\mathcal{c}_{2^{k-1}, 0}, c_{2^{k-1}, 1}, \ldots, c_{2^{k-1}, M}\right]^{T}, \\
\Psi(x)=\left[\psi_{1,0}, \ldots, \psi_{1, M}, \psi_{2,0}, \ldots, \psi_{2, M}, \ldots,\right. \\
\left.\psi_{2^{k-1}, 0}, \psi_{2^{k-1}, 1}, \ldots, \psi_{2^{k-1}, M}\right]^{T} .
\end{gathered}
$$

\section{Chebyshev Finite Difference Method}

Clenshaw and Curtis [45] introduced the following approximation of the function $f(x)$ denoted by $\left(P_{M}\right) f(x)$ as

$$
\begin{aligned}
& \left(P_{M}\right) f(x)=\sum_{m=0}^{M} f_{m} T_{m}(x), \\
& f_{m}=\frac{2}{M} \sum_{k=0}^{M} f\left(x_{k}\right) T_{m}\left(x_{k}\right),
\end{aligned}
$$

where the summation symbol with double primes denotes a sum with both the first and last terms halved. Moreover, $x_{m}$ are the extrema of the Mth-order Chebyshev polynomial $T_{M}(x)$ and are defined as

$$
x_{m}=\cos \left(\frac{m \pi}{M}\right), \quad m=0,1,2, \ldots, M
$$

These well-known Chebyshev-Gauss-Lobatto interpolated points,

$$
x_{M}=-1<x_{M-1}<\cdots<x_{1}<x_{0}=1
$$

are the zeros of $\left(1-x^{2}\right)\left(d T_{M}(x) / d x\right)$. Using (4) we have,

$$
T_{m}\left(x_{k}\right)=\cos \left(\frac{m k \pi}{M}\right) \text {, }
$$

so $f_{m}$ can be rewritten as

$$
f_{m}=\frac{2}{M} \sum_{k=0}^{M} f\left(x_{k}\right) \cos \left(\frac{m k \pi}{M}\right) .
$$


The first two derivatives of the function $f(x)$ at the points $x_{m}, m=0,1, \ldots, M$ are given by [46] as

$$
f^{(n)}\left(x_{m}\right)=\sum_{j=0}^{M} d_{m, j}^{(n)} f\left(x_{j}\right), \quad n=1,2,
$$

where

$$
\begin{aligned}
d_{m, j}^{(1)}= & \frac{4 \theta_{j}}{M} \sum_{k=1}^{M} \sum_{l=0,(k+l) \text { odd }}^{k-1} \frac{k \theta_{k}}{c_{l}} T_{k}\left(x_{j}\right) T_{l}\left(x_{m}\right) \\
= & \frac{4 \theta_{j}}{M} \sum_{k=1}^{M} \sum_{l=0,(k+l) \text { odd }}^{k-1} \frac{k \theta_{k}}{c_{l}} \\
& \times \cos \left(\frac{k j \pi}{M}\right) \cos \left(\frac{l m \pi}{M}\right), \\
d_{m, j}^{(2)}= & \frac{2 \theta_{j}}{M} \sum_{k=2}^{M} \sum_{l=0,(k+l) \text { even }}^{k-2} \frac{k\left(k^{2}-l^{2}\right) \theta_{k}}{c_{l}} T_{k}\left(x_{j}\right) T_{l}\left(x_{m}\right) \\
= & \frac{2 \theta_{j}}{M} \sum_{\mathrm{k}=2 l=0,(k+l) \text { even }}^{M} \sum_{c_{l}}^{k-2} \frac{k\left(k^{2}-l^{2}\right) \theta_{k}}{c_{l}} \cos \left(\frac{k j \pi}{M}\right) \cos \left(\frac{l m \pi}{M}\right),
\end{aligned}
$$

with $\theta_{0}=\theta_{M}=1 / 2, \theta_{j}=1$ for $j=1,2, \ldots, M-1$.

As can be seen from (24), the first two derivatives of the function $f(x)$ at any point of the Chebyshev-Gauss-Lobatto points are expanded as a linear combination of the values of the function as these points.

\section{Chebyshev Wavelet Finite Difference Method}

In this section, we present the Chebyshev wavelet finite difference (CWFD) method. Consider $x_{n m}, n=1,2, \ldots, 2^{k-1}$, $m=0,1, \ldots, M$, as the corresponding Chebyshev-GaussLobatto collocation points at the $n$th subinterval $[(n-$ 1) $/ 2^{(k-1)}, n / 2^{(k-1)}$ ] such that

$$
x_{n m}=\frac{1}{2^{k}}\left(x_{m}+2 n-1\right) \text {. }
$$

A function $f(x)$ can be written in terms of Chebyshev wavelet basis functions as follows:

$$
\left(P_{M}\right) f(x)=\sum_{n=1}^{2^{k-1}} \sum_{m=0}^{M}{ }^{\prime \prime} c_{n m} \psi_{n m}(x),
$$

where $c_{n m}, n=1,2, \ldots, 2^{k-1}, m=0,1, \ldots, M$, are the expansion coefficients of the function $f(x)$ at the subinterval $\left[(n-1) / 2^{k-1}, n / 2^{k-1}\right]$ and $\psi_{n, m}(x), n=1,2, \ldots, 2^{k-1}, m=$ $0,1, \ldots, M$, are defined in (11).
In view of (11) and (19), we can obtain the coefficients $c_{n m}$ as

$$
\begin{aligned}
c_{n m} & =\frac{1}{\left(2^{k / 2} p_{m}\right)^{2}} \cdot \frac{2}{M} \sum_{p=0}^{M} f\left(x_{n p}\right) \psi_{n m}\left(x_{n p}\right) \\
& =\frac{1}{2^{k / 2} p_{m}} \cdot \frac{2}{M} \sum_{p=0}^{M} f\left(x_{n p}\right) \cos \left(\frac{m p \pi}{M}\right) .
\end{aligned}
$$

Using (25), the first two derivatives of the function $f(x)$ at the points $x_{n m}, n=1,2, \ldots, 2^{k-1}, m=0,1, \ldots, M$, can be obtained as

$$
f^{(n)}\left(x_{n m}\right)=\sum_{j=0}^{M} d_{n, m, j}^{(n)} f\left(x_{n j}\right), \quad n=1,2,
$$

where

$$
\begin{aligned}
& d_{n, m, j}^{(1)}=\frac{4 \theta_{j}}{M} \sum_{k=1}^{M} \sum_{l=0,(k+l) \text { odd }}^{k-1} \frac{k \theta_{k}}{c_{l} p_{k} p_{l}} \psi_{n k}\left(x_{n j}\right) \psi_{n l}\left(x_{n m}\right) \\
&=\frac{4 \theta_{j}}{M} \sum_{k=1}^{M} \sum_{l=0,(k+l) \text { odd }}^{k-1} \frac{2^{k} k \theta_{k}}{c_{l}} \cos \left(\frac{k j \pi}{M}\right) \cos \left(\frac{l m \pi}{M}\right), \\
& d_{m, j}^{(2)}=\frac{2 \theta_{j}}{M} \sum_{k=2}^{M} \sum_{l=0,(k+l) \text { even }}^{k-2} \frac{2^{k} k\left(k^{2}-l^{2}\right) \theta_{k}}{c_{l} p_{k} p_{l}} \\
&=\frac{2 \theta_{j}}{M} \sum_{k=2}^{M} \sum_{l=0,(k+l) \text { even }}^{k-2} \frac{4^{k} k\left(k^{2}-l^{2}\right) \theta_{k}}{c_{l}} \\
& \times \cos \left(\frac{k j \pi}{M}\right) \cos \left(\frac{l m \pi}{M}\right) .
\end{aligned}
$$

\section{Convergence Analysis}

Lemma 5. If the Chebyshev wavelet expansion of a continuous function $f(x)$ converges uniformly, then the Chebyshev wavelet expansion converges to the function $f(x)$ [47].

Theorem 6. A function $f(x) \in L_{w_{n}}^{2}[0,1)$, with bounded second derivative, say $\left|f^{\prime \prime}(x)\right| \leq B$, can be expanded as an infinite sum of Chebyshev wavelets, and the series converges uniformly to $f(x)$; that is,

$$
f(x)=\sum_{n=1}^{\infty} \sum_{m=0}^{\infty} \widehat{c}_{n m} \psi_{n m}(x) .
$$

Proof. We have

$$
\begin{gathered}
\widehat{c}_{n, m}=\left(f(x), \psi_{n, m}(x)\right)_{w_{n, k}}=\int_{0}^{1} f(x) \psi_{n m}(x) w_{n, k}(x) d x \\
=\int_{(n-1) / 2^{(k-1)}}^{n / 2^{(k-1)}} 2^{k / 2} p_{m} f(x) T_{m}\left(2^{k} x-2 n+1\right) w \\
\quad \times\left(2^{k} x-2 n+1\right) d x
\end{gathered}
$$


if $m>1$, by substituting $2^{k} x-2 n+1=\cos \alpha$, it yields

$$
\widehat{c}_{n m}=\frac{1}{2^{k / 2}} \int_{0}^{\pi} f\left(\frac{\cos \alpha+2 n-1}{2^{k}}\right) \sqrt{\frac{2}{\pi}} \cos m \alpha d \alpha .
$$

Using integration by part, we get

$$
\begin{aligned}
\widehat{c}_{n m}= & \left.\frac{\sqrt{2}}{2^{k / 2} \sqrt{\pi}} f\left(\frac{\cos \alpha+2 n-1}{2^{k}}\right)\left(\frac{\sin m \alpha}{m}\right)\right|_{0} ^{\pi} \\
& +\frac{\sqrt{2}}{2 \frac{3 k}{2} m \sqrt{\pi}} \int_{0}^{\pi} f^{\prime}\left(\frac{\cos \alpha+2 n-1}{2^{k}}\right) \sin m \alpha \sin \alpha d \alpha ;
\end{aligned}
$$

the first part is zero, therefore,

$$
\widehat{c}_{n m}=\frac{\sqrt{2}}{2^{3 k / 2} m \sqrt{\pi}} \int_{0}^{\pi} f^{\prime}\left(\frac{\cos \alpha+2 n-1}{2^{k}}\right) \sin m \alpha \sin \alpha d \alpha .
$$

Using integration by part again, it yields

$$
\begin{aligned}
\widehat{c}_{n m}= & \frac{1}{2^{3 k / 2} m \sqrt{2 \pi}} f^{\prime}\left(\frac{\cos \alpha+2 n-1}{2^{k}}\right) \\
& \times\left.\left(\frac{\sin (m-1) \alpha}{m-1}-\frac{\sin (m+1) \alpha}{m+1}\right)\right|_{0} ^{\pi} \\
& +\frac{1}{2^{5 k / 2} m \sqrt{2 \pi}} \int_{0}^{\pi} f^{\prime \prime}\left(\frac{\cos \alpha+2 n-1}{2^{k}}\right) r_{m}(\alpha) d \alpha
\end{aligned}
$$

where

$$
r_{m}(\alpha)=\sin \alpha\left(\frac{\sin (m-1) \alpha}{m-1}-\frac{\sin (m+1) \alpha}{m+1}\right) .
$$

Thus, we get

$$
\begin{aligned}
\left|\widehat{c}_{n m}\right| & =\left|\frac{1}{2^{5 k / 2} m \sqrt{2 \pi}} \int_{0}^{\pi} f^{\prime \prime}\left(\frac{\cos \alpha+2 n-1}{2^{k}}\right) r_{m}(\alpha) d \alpha\right| \\
& \leq\left(\frac{1}{2^{5 k / 2} m \sqrt{2 \pi}}\right) \int_{0}^{\pi}\left|f^{\prime \prime}\left(\frac{\cos \alpha+2 n-1}{2^{k}}\right) r_{m}(\alpha)\right| d \alpha \\
& \leq \frac{B}{2^{5 k / 2} m \sqrt{2 \pi}} \int_{0}^{\pi}\left|r_{m}(\alpha)\right| d \alpha .
\end{aligned}
$$

However

$$
\begin{aligned}
\int_{0}^{\pi}\left|r_{m}(\alpha)\right| d \alpha & =\int_{0}^{\pi}\left|\sin \alpha\left(\frac{\sin (m-1) \alpha}{m-1}-\frac{\sin (m+1) \alpha}{m+1}\right)\right| d \alpha \\
\leq & \int_{0}^{\pi}\left|\frac{\sin \alpha \sin (m-1) \alpha}{m-1}\right| \\
& +\left|\frac{\sin \alpha \sin (m+1) \alpha}{m+1}\right| d \alpha \leq \frac{2 m \pi}{m^{2}-1} .
\end{aligned}
$$

Since $n \leq 2^{k-1}$, we obtain

$$
\left|\widehat{c}_{n m}\right| \leq \frac{\sqrt{2 \pi} B}{(2 n)^{5 / 2}\left(m^{2}-1\right)} .
$$

Now, if $m=1$, by using (35), we have

$$
\left|\widehat{c}_{n 1}\right|<\frac{\sqrt{2 \pi}}{(2 n)^{3 / 2}} \max _{0 \leq x \leq 1}\left|f^{\prime}(x)\right| \text {. }
$$

It is mentioned in [42] that $\left\{\psi_{n 0}\right\}_{n=1}^{\infty}$ form an orthogonal system constructed by Haar scaling function with respect to the weight function $w(x)$, so $\sum_{n=1}^{\infty}\left(2^{k} / \sqrt{ } \pi\right) \widehat{c}_{n 0} \psi_{n 0}(x)$ is convergent. Hence, we will have

$$
\begin{aligned}
\left|\sum_{n=1}^{\infty} \sum_{m=0}^{\infty} \widehat{c}_{n m} \psi_{n m}(x)\right| \leq & \left|\frac{2^{k}}{\sqrt{\pi}} \sum_{n=1}^{\infty} \widehat{c}_{n 0} \psi_{n 0}(x)\right| \\
& +\sum_{n=1}^{\infty} \sum_{m=1}^{\infty}\left|\widehat{c}_{n m}\right|\left|\psi_{n m}(x)\right| \\
\leq & \left|\frac{2^{k}}{\sqrt{\pi}} \sum_{n=1}^{\infty} \widehat{c}_{n 0} \psi_{n 0}(x)\right| \\
& +\frac{2}{\frac{(k+1)}{2}} \sum_{n=1}^{\infty} \sum_{m=1}^{\infty}\left|\widehat{c}_{n m}\right|<\infty .
\end{aligned}
$$

Therefore, with the aid of Lemma 5 , the series $\sum_{n=1}^{\infty} \sum_{m=1}^{\infty}$ $\widehat{c}_{n m} \psi_{n m}(x)$ converges to $f(x)$ uniformly [47].

Theorem 7. Suppose $f(x) \in L_{w_{n}}^{2}[0,1)$ with bounded second derivative, say $\left|f^{\prime \prime}(t)\right| \leq B$; then its Chebyshev wavelet finite difference expansion converges uniformly to $f(x)$; that is,

$$
\sum_{n=1}^{2^{k-1}} \sum_{m=0}^{\prime} c_{n m} \psi_{n m}(x)=f(x)
$$

where the summation symbol with prime denotes a sum with the first term halved.

Proof. From Theorem 6, we have

$$
f(x)=\sum_{n=1}^{2^{k-1}} \sum_{m=0}^{\infty} \widehat{c}_{n m} \psi_{n m}(x),
$$

where

$$
\widehat{c}_{n, m}=\left(f(x), \psi_{n, m}(x)\right)_{w_{n}} .
$$

This series converges to $f(x)$ uniformly. We first show that $c_{n m}$ converges to $\widehat{c}_{n m}$. We have

$$
\begin{aligned}
\widehat{c}_{n, m}= & \left(f(x), \psi_{n, m}(x)\right)_{w_{n, k}}=\int_{0}^{1} f(x) \psi_{n m}(x) w_{n, k}(x) d x \\
= & \int_{(n-1) / 2^{(k-1)}}^{n / 2^{(k-1)}} 2^{k / 2} p_{m} f(x) T_{m}\left(2^{k} x-2 n+1\right) \\
& \times w\left(2^{k} x-2 n+1\right) d x
\end{aligned}
$$


by substituting $2^{k} x-2 n+1=\cos \alpha$, it yields

$$
\widehat{c}_{n m}=\frac{p_{m}}{2^{k / 2}} \int_{0}^{\pi} f\left(\frac{\cos \alpha+2 n-1}{2^{k}}\right) \cos m \alpha d \alpha
$$

Using the trapezoidal rule for integration with $M$ equidistant subintervals gives

$$
\begin{array}{r}
\widehat{c}_{n m} \simeq \frac{p_{m}}{2^{k / 2}} \cdot \frac{\pi}{M} \sum_{j=0}^{M} f\left(\frac{\cos \left(\alpha_{j}\right)+2 n-1}{2^{k}}\right) \\
\times \cos \left(m \alpha_{j}\right), \quad \alpha_{j}=\frac{j \pi}{M} .
\end{array}
$$

From (26) and (28), for $1<m<M$, we have

$$
\widehat{c}_{n m} \simeq \frac{1}{2^{k / 2} p_{m}} \cdot \frac{2}{M} \sum_{j=0}^{M \prime \prime} f\left(x_{n j}\right) \cos \left(\frac{m j \pi}{M}\right)=c_{n m} .
$$

According to approximation error for the trapezoidal rule, we have

$$
\left|\widehat{c}_{n m}-c_{n m}\right| \leq \frac{R \pi^{3}}{12 M^{2}}
$$

where

$$
\begin{gathered}
R=\max \left\{\frac{d^{2}}{d \alpha^{2}}\left(f\left(\frac{\cos (\alpha)+2 n-1}{2^{k}}\right) \cos (m \alpha)\right),\right. \\
0 \leq \alpha \leq \pi\} .
\end{gathered}
$$

In view of (35) and triangle inequality, we get

$$
\begin{aligned}
\left|c_{n m}\right| & \leq\left|c_{n m}-\widehat{c}_{n m}\right|+\left|\widehat{c}_{n m}\right| \\
& \leq \frac{R \pi^{3}}{12 M^{2}}+\frac{\sqrt{2 \pi} B}{(2 n)^{5 / 2}\left(m^{2}-1\right)} \\
& \leq \frac{R \pi^{3}}{12\left(m^{2}-1\right)}+\frac{\sqrt{2 \pi} B}{(2 n)^{5 / 2}\left(m^{2}-1\right)} \\
& \leq \frac{C}{\left(m^{2}-1\right)},
\end{aligned}
$$

where

$$
C=\frac{R \pi^{3}}{12}+\frac{\sqrt{2 \pi} B}{(2 n)^{5 / 2}}
$$

Because $\left|\psi_{n m}(x)\right| \leq 2^{k / 2} p_{m}$, it is understandable that

$$
\begin{aligned}
\left|\sum_{n=1}^{2^{k-1}} \sum_{m=0}^{\infty}{ }^{\prime} c_{n m} \psi_{n m}(x)\right| & \left|\sum_{n=1}^{2^{k-1}} \frac{1}{2} c_{n 0} \psi_{n 0}(x)\right|+\left|\sum_{n=1}^{2^{k-1}} c_{n 1} \psi_{n 1}(x)\right| \\
& +\sum_{n=1}^{2_{m=2}^{k-1}}\left|c_{n m}\right|\left|\psi_{n m}(x)\right| \\
\leq & \frac{2^{k / 2}}{2 \sqrt{\pi}} \sum_{n=1}^{2^{k-1}}\left|c_{n 0}\right|+\frac{2^{(k+1) / 2}}{\sqrt{\pi}} \sum_{n=1}^{2^{k-1}}\left|c_{n 1}\right|+\frac{2^{(k+1) / 2}}{\sqrt{\pi}} \\
& \times \sum_{n=1}^{2^{k-1}} \sum_{m=2}^{\infty}\left|c_{n m}\right|<\infty .
\end{aligned}
$$

Therefore, in view of Lemma 5, series (43) is uniformly convergent to $f(x)$.

Theorem 8 (accuracy estimation). Suppose $f(x) \in L_{w_{n}}^{2}[0,1)$ with bounded second derivative, say $\left|f^{\prime \prime}(x)\right| \leq B$, then one has the following accuracy estimation:

$$
\sigma_{k, M} \leq\left(\sum_{n=1}^{2^{k-1}} \sum_{m=M+1}^{\infty} \frac{C^{2}}{\left(m^{2}-1\right)^{2}}\right)^{1 / 2}
$$

where

$$
\begin{gathered}
\sigma_{k, M}=\left(\int_{0}^{1}\left[f(x)-\sum_{n=1}^{2^{k-1}} \sum_{m=0}^{M} c_{n m} \psi_{n, m}(x)\right]^{2} w_{n}(x) d x\right)^{1 / 2}, \\
C=\frac{R \pi^{3}}{12}+\frac{\sqrt{2 \pi} B}{(2 n)^{5 / 2}} .
\end{gathered}
$$

Proof. We have

$$
\begin{aligned}
\sigma_{k, M}^{2}= & \int_{0}^{1}\left[f(x)-\sum_{n=1}^{2^{k-1}} \sum_{m=0}^{M} c_{n m} \psi_{n, m}(x)\right]^{2} w_{n}(x) d x \\
= & \int_{0}^{1}\left[\sum_{n=1}^{2^{k-1}} \sum_{m=0}^{\infty} c_{n m} \psi_{n, m}(x)\right. \\
& \left.-\int_{0=1}^{1} \sum_{m=0}^{1} c_{n m}^{2^{k-1}} \psi_{n, m}(x)\right]^{2} w_{n}(x) d x \\
= & \sum_{n=1}^{2}\left(\psi_{n, m}(x)\right)^{2} w_{n}(x) d x \\
2^{k-1} & \sum_{m=M+1}^{\infty} c_{n m}^{2} \int_{0}^{1}\left(\psi_{n, m}(x)\right)^{2} w_{n}(x) d x .
\end{aligned}
$$


We know that the family $\left\{\psi_{n, m}(x) \mid n=1,2, \ldots, 2^{k-1}, m \in\right.$ $\mathbb{N} \cup\{0\}\}$ forms orthonormal basis for $L_{w_{n}}^{2}[0,1)$, so $\int_{0}^{1}\left(\psi_{n, m}(x)\right)^{2} w_{n}(x) d x=1$. Therefore, in view of (52), we will have

$$
\sigma_{k, M}^{2}=\sum_{n=1}^{2^{k-1}} \sum_{m=M+1}^{\infty} c_{n m}^{2} \leq \sum_{n=1}^{2^{k-1}} \sum_{m=M+1}^{\infty} \frac{C^{2}}{\left(m^{2}-1\right)^{2}}
$$

where

$$
C=\frac{R \pi^{3}}{12}+\frac{\sqrt{2 \pi} B}{(2 n)^{5 / 2}}
$$

\section{Discretization of Problem}

In this section, the Chebyshev wavelet finite difference method (CWFD) is used for solving the following general form:

$$
\begin{array}{r}
H\left(t, y(t), D^{\beta_{1}} y(t), \ldots, D^{\beta_{k}} y(t), \ldots, D^{\beta_{p}} y(t)\right)=0, \\
t \in I=[0, L],
\end{array}
$$

subject to the conditions

$$
\begin{gathered}
B_{q}\left(y\left(\rho_{0}\right), \ldots, y^{(l)}\left(\rho_{0}\right), y\left(\rho_{1}\right), \ldots, y^{(l)}\left(\rho_{1}\right), \ldots,\right. \\
\left.y\left(\rho_{l}\right), \ldots, y^{(l)}\left(\rho_{l}\right)\right) \\
q=0,1, \ldots, l
\end{gathered}
$$

where $0<\beta_{1}<\beta_{2}<\cdots<\beta_{p}, l<\beta_{p} \leq l+1, \rho_{j}, j=0,1, \ldots, l$ are located in $[0, L]$ and $H$ can be linear or nonlinear while $B_{q}$ are linear functions.

We suppose the interval $[0,1]$ is divided into $2^{k-1}$ subintervals $I_{n}=\left[(n-1) / 2^{k-1}, n / 2^{k-1}\right], n=1,2, \ldots, 2^{k-1}$. We also consider the shifted Chebyshev-Gauss-Lobatto collocation points $(n-1) / 2^{k-1}=t_{n 0}<t_{n 1}<\cdots<t_{n, M-1}<t_{n, M}=n / 2^{k-1}$ on the $n$th subinterval $I_{n}, n=1,2, \ldots, 2^{k-1}$, where $t_{n s}$ is defined as follows:

$$
t_{n s}=\frac{1}{2^{k}}\left(t_{s}+2 n-1\right), \quad s=1,2, \ldots, M-l .
$$

In order to obtain the solution $y(t)$ in $(60)$, we first approximate $y(t)$ according to $(27)$ and rewrite $D^{\beta_{i}} y(t), i=$ $1, \ldots, p$ as fractional derivatives of $y(t)$, in the Caputo sense using (2). Collocating (60) at the shifted Chebyshev-GaussLobatto points $t_{n s}, n=1,2, \ldots, 2^{k-1}, s=0,1, \ldots, M-l$, we get

$$
\begin{aligned}
& H\left(t_{n s}, y\left(t_{n s}\right), D^{\beta_{1}} y\left(t_{n s}\right), \ldots,\right. \\
& \left.D^{\beta_{k}} y\left(t_{n s}\right), \ldots, D^{\beta_{p}} y\left(t_{n s}\right)\right)=0 .
\end{aligned}
$$

We then continue as follows:

$$
\begin{array}{rl}
D^{\beta_{i}} & y\left(t_{n s}\right) \\
= & \frac{1}{\Gamma\left(n_{i}-\beta_{i}\right)} \int_{0}^{t_{n s}}\left(t_{n s}-\tau\right)^{n_{i}-\beta_{i}-1} y^{\left(n_{i}\right)}(\tau) d \tau \\
n_{i}=\left[\beta_{i}\right]+1, i=1, \ldots, p \\
=\frac{1}{\Gamma\left(n_{i}-\beta_{i}\right)} \int_{0}^{(n-1) / 2^{(k-1)}}\left(t_{n s}-\tau\right)^{n_{i}-\beta_{i}-1} y^{\left(n_{i}\right)}(\tau) d \tau \\
\quad+\frac{1}{\Gamma\left(n_{i}-\beta_{i}\right)} \int_{(n-1) / 2^{(k-1)}}^{t_{n s}}\left(t_{n s}-\tau\right)^{n_{i}-\beta_{i}-1} y^{\left(n_{i}\right)}(\tau) d \tau .
\end{array}
$$

To calculate the first integral in the above summation, we use the Clenshaw-Curtis quadrature formula [48]:

$$
\int_{-1}^{1} f(x) d x=\sum_{r=0}^{N} w_{r} f\left(x_{r}\right)
$$

where $x_{r}$ are Chebyshev-Gauss-Lobatto nodes and the weights $w_{r}$ are given by

$$
\begin{gathered}
w_{0}=w_{N}= \begin{cases}\frac{1}{N^{2}}, & N \text { odd, } \\
\frac{1}{N^{2}-1}, & N \text { even, }\end{cases} \\
w_{r}=\frac{2}{N \gamma_{r}}\left[1-\sum_{k=1}^{[N / 2]} \frac{2}{\gamma_{2 k}\left(4 k^{2}-1\right)} \cos \frac{2 k r \pi}{N}\right], \\
r=1,2, \ldots, N-1,
\end{gathered}
$$

where $\gamma_{0}=\gamma_{N}=2$ and $\gamma_{r}=1$, for $r=1,2, \ldots, N-1$.

In this paper we set $N=M$. We obtain

$$
\begin{aligned}
& \frac{1}{\Gamma\left(n_{i}-\beta_{i}\right)} \int_{0}^{(n-1) / 2^{k-1}}\left(t_{n s}-\tau\right)^{n_{i}-\beta_{i}-1} y^{\left(n_{i}\right)}(\tau) d \tau \\
& =\frac{1}{\Gamma\left(n_{i}-\beta_{i}\right)} \\
& \quad \times \sum_{r_{1}=1}^{n-1} \int_{\left(r_{1}-1\right) / 2^{k-1}}^{r_{1} / 2^{k-1}}\left(t_{n s}-\tau\right)^{n_{i}-\beta_{i}-1} y^{\left(n_{i}\right)}(\tau) d \tau \\
& =\frac{1}{\Gamma\left(n_{i}-\beta_{i}\right)} \cdot \frac{1}{2^{k}} \sum_{r_{1}=1}^{n-1} \sum_{r_{2}=0}^{N} w_{r_{2}}\left(t_{n s}-t_{r_{1} r_{2}}\right)^{n_{i}-\beta_{i}-1} y^{\left(n_{i}\right)}\left(t_{r_{1} r_{2}}\right)
\end{aligned}
$$


Using integration by part and in view of (62), we convert second singular integral to nonsingular one as follows: get the second integral

$$
\begin{gathered}
\frac{1}{\Gamma\left(n_{i}-\beta_{i}\right)} \int_{(n-1) / 2^{k-1}}^{t_{n s}}\left(t_{n s}-\tau\right)^{n_{i}-\beta_{i}-1} y^{\left(n_{i}\right)}(\tau) d \tau \\
=\frac{\left(t_{n s}-\left((n-1) / 2^{k-1}\right)\right)^{n_{i}-\beta_{i}}}{\left(n_{i}-\beta_{i}\right) \Gamma\left(n_{i}-\beta_{i}\right)} y^{\left(n_{i}\right)}\left(\frac{n-1}{2^{k-1}}\right) \\
+\frac{\left(2^{k-1} t_{n s}-n+1\right)}{2^{k}\left(n_{i}-\beta_{i}\right) \Gamma\left(n_{i}-\beta_{i}\right)}, \\
\sum_{s_{1}=0}^{N}\left(t_{n s}-\left(t_{n s}-\frac{n-1}{2^{k-1}}\right)\left(2^{k-1} t_{n s_{1}}-n+1\right)-\frac{n-1}{2^{k-1}}\right)^{n_{i}-\beta_{i}} \\
y^{\left(n_{i}+1\right)}\left(\left(t_{n s}-\frac{n-1}{2^{k-1}}\right)\left(2^{k-1} t_{n s_{1}}-n+1\right)+\frac{n-1}{2^{k-1}}\right) .
\end{gathered}
$$

Replacing (67) and (68) into (64), we obtain $D^{\beta_{i}} y\left(t_{n s}\right), i=$ $1,2, \ldots, p$ and then replace them into (64) to get $2^{k-1}(M-l)$ equations.

Furthermore, substituting (27) and (29) into (61), we get $(l+1)$ equations.

Moreover, we should impose continuity condition on the approximate solution and its first $l$ derivatives at the interface between subintervals which results in $\left(2^{k-1}-1\right)(l+1)$ equations.

$$
\begin{array}{r}
y^{(r)}\left(t_{n+1, M}\right)=y^{(r)}\left(t_{n, 0}\right), \\
r=0,1, \ldots, l, n=1,2, \ldots, 2^{k-1}-1 .
\end{array}
$$

We will totally have a system of $2^{k-1}(M+1)$ algebraic equations, which can be solved for the $y\left(t_{n s}\right)$. Consequently, we obtain the solution $y(t)$ to the given (60) using (27) and (28).

\section{Illustrative Examples}

In this section, we consider some numerical examples for the fractional equation to demonstrate the validity of the proposed method (CWFD) in solving fractional differential equation. These examples are considered because closed form solutions are available for them, and they have been solved using other numerical methods. This allows one to compare the results obtained using this method with the analytical solution and the solutions obtained using other methods.

Example 1. As the first example, we consider a nonlinear equation defined as follows $[49,50]$ :

$$
\begin{aligned}
D^{\alpha} y(t)= & \frac{40320}{\Gamma(9-\alpha)} t^{8-\alpha}-3 \frac{\Gamma(5+\alpha / 2)}{\Gamma(5-\alpha / 2)} t^{4-(\alpha / 2)} \\
& +\frac{9}{4} \Gamma(\alpha+1)+\left(\frac{3}{2} t^{(\alpha / 2)}-t^{4}\right)^{3}-[y(t)]^{(3 / 2)},
\end{aligned}
$$

such that

$$
y(0)=0, \quad y^{\prime}(0)=0
$$

the second initial condition is for $\alpha>1$ only. The exact solution of (70) and (71) is given as [26]

$$
y(t)=t^{8}-3 t^{4+\alpha / 2}+\frac{9}{4} t^{\alpha}
$$

It should be noted that for $\alpha<1$, the slope of the solution at $t=0$ goes to infinity. Therefore, one can expect a large numerical error near $t=0$.

We applied the method introduced in Section 6 and solved this problem for different values of $\alpha$ and $M, k$. Figure 1 shows the analytical and numerical results for $M=9,6,3$ and $k=1$ and $\alpha=0.75,1.5$. It can be seen that increasing the values of $M$ and $k$ results in more accurate solution. In Figure 2 numerical results for $M=12, k=4$, and $\alpha=0.5,0.75,0.95$ and $\alpha=1$ (exact solution) and also $\alpha=1.5,1.75,1.95$, and $\alpha=2$ (exact solution) are plotted. We see that as $\alpha$ approaches 1 and 2, the solution of the fractional differential equation approaches to that of the integer-order differential equation. In Table 1, we compare the results obtained by the present method using $M=12, k=6$ with those in [50]. As can be seen, our results are much more better than those obtained by Saadatmandi and Dehghan [50]. Furthermore, as it is expected, the absolute error is reduced as $\alpha$ approaches an integer value.

Example 2. As the second example, we consider the following initial value problem in the case of the inhomogeneous Bagley-Torvik equation [51]:

$$
D^{2} y(t)+D^{1.5} y(t)+y(t)=g(t)
$$

where $g(t)=1+t$ subject to the following initial states

$$
y(0)=1, \quad y^{\prime}(0)=1 \text {. }
$$

The exact solution of this problem is $y(t)=1+t$.

We solve this fractional initial value problem by applying the method described in Section 6 with $M=k=3$. The absolute error is shown in Figure 3, which shows that the numerical solution is in perfect agreement with the exact solution. We set Digits $=50$. However, we get the absolute error less or equal to $10^{-18}$ when we set Digits $=20$.

Example 3. Following El-Mesiry et al. [52] and Li [33], we consider the following nonlinear fractional differential equation:

$$
\begin{array}{r}
a D^{2.0} y(t)+b D^{\alpha_{2}} y(t)+c D^{\alpha_{1}} y(t)+e[y(t)]^{3}=f(t), \\
0<\alpha_{1} \leq 1,1<\alpha_{2} \leq 2,
\end{array}
$$

where

$$
f(t)=\frac{2 a}{\Gamma(2)} t+\frac{2 b}{\Gamma\left(4-\alpha_{2}\right)} t^{3-\alpha_{2}}+\frac{2 c}{\Gamma\left(4-\alpha_{1}\right)} t^{3-\alpha_{1}}+e\left[\frac{1}{3} t^{3}\right]^{3},
$$

subject to

$$
y(0)=y^{\prime}(0)=0 .
$$

The exact solution of this problem is $y(t)=(1 / 3) t^{3}$. 
TABLE 1: Comparison of absolute error in $y(t)$ in [50] and obtained using our method for Example 1.

\begin{tabular}{|c|c|c|c|c|c|c|c|c|}
\hline \multirow{2}{*}{$\alpha$} & \multicolumn{2}{|c|}{$t=0.1$} & \multicolumn{2}{|c|}{$t=0.3$} & \multicolumn{2}{|c|}{$t=0.7$} & \multicolumn{2}{|c|}{$t=0.9$} \\
\hline & Our result & Ref. [50] & Our result & Ref. [50] & Our result & Ref. [50] & Our result & Ref. [50] \\
\hline 0.2 & $8.6 \times 10^{-5}$ & $2.2 \times 10^{-1}$ & $2.6 \times 10^{-5}$ & $2.3 \times 10^{-1}$ & $1.1 \times 10^{-5}$ & $5.3 \times 10^{-1}$ & $1.0 \times 10^{-5}$ & $1.7 \times 10^{0}$ \\
\hline 0.4 & $1.7 \times 10^{-4}$ & $6.3 \times 10^{-2}$ & $5.3 \times 10^{-5}$ & $6.0 \times 10^{-2}$ & $2.0 \times 10^{-5}$ & $1.2 \times 10^{-1}$ & $1.8 \times 10^{-5}$ & $3.0 \times 10^{-1}$ \\
\hline 0.6 & $1.9 \times 10^{-4}$ & $1.5 \times 10^{-2}$ & $7.5 \times 10^{-5}$ & $1.3 \times 10^{-2}$ & $3.0 \times 10^{-5}$ & $2.1 \times 10^{-2}$ & $2.7 \times 10^{-5}$ & $3.7 \times 10^{-2}$ \\
\hline 0.8 & $1.2 \times 10^{-4}$ & $2.9 \times 10^{-3}$ & $7.1 \times 10^{-5}$ & $2.1 \times 10^{-3}$ & $4.8 \times 10^{-5}$ & $2.5 \times 10^{-3}$ & $5.2 \times 10^{-5}$ & $2.1 \times 10^{-3}$ \\
\hline 1.2 & $5.1 \times 10^{-3}$ & $1.9 \times 10^{-3}$ & $1.8 \times 10^{-2}$ & $1.6 \times 10^{-3}$ & $3.5 \times 10^{-2}$ & $2.9 \times 10^{-3}$ & $4.0 \times 10^{-2}$ & $1.6 \times 10^{-2}$ \\
\hline 1.4 & $2.8 \times 10^{-4}$ & $2.0 \times 10^{-4}$ & $1.3 \times 10^{-3}$ & $1.6 \times 10^{-3}$ & $2.8 \times 10^{-3}$ & $4.9 \times 10^{-3}$ & $3.3 \times 10^{-3}$ & $3.3 \times 10^{-2}$ \\
\hline 1.6 & $4.3 \times 10^{-5}$ & $6.3 \times 10^{-5}$ & $3.2 \times 10^{-5}$ & $7.3 \times 10^{-4}$ & $2.9 \times 10^{-5}$ & $2.3 \times 10^{-3}$ & $6.0 \times 10^{-5}$ & $1.3 \times 10^{-2}$ \\
\hline 1.8 & $3.0 \times 10^{-5}$ & $3.8 \times 10^{-5}$ & $7.3 \times 10^{-5}$ & $2.0 \times 10^{-4}$ & $1.5 \times 10^{-4}$ & $5.9 \times 10^{-4}$ & $1.9 \times 10^{-4}$ & $2.8 \times 10^{-3}$ \\
\hline
\end{tabular}

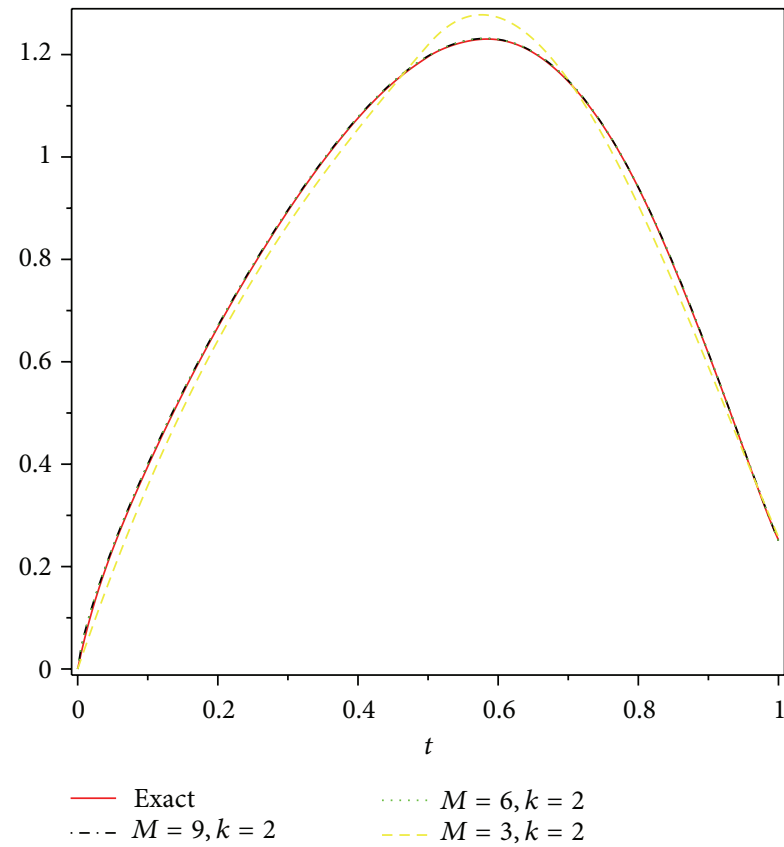

(a)

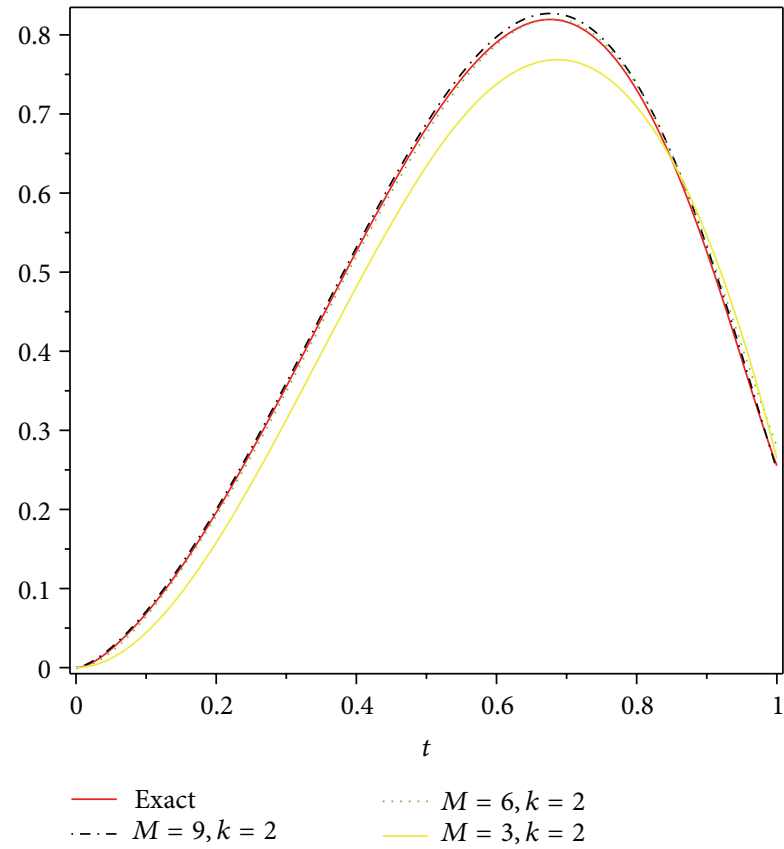

(b)

Figure 1: Comparison of $y(t)$ for $M=9,6,3$ and $k=2$ and (a) $\alpha=0.75$, (b) $\alpha=1.5$, with exact solution, for Example 1 .

TABle 2: Comparison of absolute error in solution of our method and reported in [33] for Example 3.

\begin{tabular}{lcc}
\hline$t$ & Our method & Chebyshev wavelets method [33] \\
& $M=9, k=3$ & $m 84$ \\
\hline 0.1 & $4.318636 \times 10^{-10}$ & $3.260279 \times 10^{-7}$ \\
0.2 & $6.606933 \times 10^{-9}$ & $7.929112 \times 10^{-7}$ \\
0.3 & $4.151355 \times 10^{-8}$ & $1.158235 \times 10^{-6}$ \\
0.4 & $9.317364 \times 10^{-8}$ & $1.184173 \times 10^{-6}$ \\
0.5 & $1.561308 \times 10^{-7}$ & $1.981458 \times 10^{-6}$ \\
0.6 & $2.914365 \times 10^{-7}$ & $1.681103 \times 10^{-6}$ \\
0.7 & $4.226536 \times 10^{-7}$ & $2.482836 \times 10^{-6}$ \\
0.8 & $5.374743 \times 10^{-7}$ & $2.783712 \times 10^{-6}$ \\
0.9 & $3.388977 \times 10^{-6}$ & $2.343684 \times 10^{-6}$ \\
\hline
\end{tabular}

For $a=b=c=e=1, \alpha_{1}=0.333, \alpha_{2}=1.234$, in Table 2, we compare the absolute errors in the solution with those obtained using Chebyshev wavelet method [33]. It can be seen that our results are much more accurate. We also show absolute errors for different values of $M$ and $k$ in Table 3. It is observed that the absolute errors in solution are reduced by increasing the values of $M$ and $k$.

Example 4. Consider the following boundary value problem in the case of the inhomogeneous Bagley-Torvik equation [53, 54]

$$
\begin{array}{r}
D^{2} y(t)+D^{3 / 2} y(t)+y(t)=t^{2}+4 \sqrt{\frac{t}{\pi}}+2, \\
y(0)=0, \quad y(50)=2500,
\end{array}
$$

where the exact solution is $y(t)=t^{2}$.

It is worth mentioning that the method presented above only can be employed for solving this problem for $t \in[0,1]$. That is because the first kind Chebyshev wavelet is defined on interval $[0,1]$. However, the variable $t$ in this example is defined on interval $[0,50]$, and we should replace Chebyshev 
TABLE 3: Obtained absolute errors for different values of $M$ and $k$ for Example 3.

\begin{tabular}{lcccc}
\hline$t$ & $M=9, k=3$ & $M=12, k=3$ & $M=15, k=3$ & $M=20, k=4$ \\
\hline 0.1 & $4.318636 \times 10^{-10}$ & $1.665675 \times 10^{-10}$ & $7.328301 \times 10^{-11}$ & $6.046732 \times 10^{-11}$ \\
0.2 & $6.606933 \times 10^{-9}$ & $2.590659 \times 10^{-9}$ & $1.134788 \times 10^{-9}$ & $8.408221 \times 10^{-10}$ \\
0.3 & $4.151355 \times 10^{-8}$ & $1.580848 \times 10^{-8}$ & $7.193868 \times 10^{-9}$ & $2.628054 \times 10^{-9}$ \\
0.4 & $9.317364 \times 10^{-8}$ & $3.453152 \times 10^{-8}$ & $1.573303 \times 10^{-8}$ & $5.658458 \times 10^{-9}$ \\
0.5 & $1.561308 \times 10^{-7}$ & $5.795390 \times 10^{-8}$ & $2.634232 \times 10^{-8}$ & $1.014053 \times 10^{-8}$ \\
0.6 & $2.914365 \times 10^{-7}$ & $1.074635 \times 10^{-7}$ & $4.947062 \times 10^{-8}$ & $1.724892 \times 10^{-8}$ \\
0.7 & $4.226536 \times 10^{-7}$ & $1.550796 \times 10^{-7}$ & $7.144234 \times 10^{-7}$ & $2.685408 \times 10^{-8}$ \\
0.8 & $5.374743 \times 10^{-7}$ & $1.810074 \times 10^{-7}$ & $7.570217 \times 10^{-8}$ & $3.913804 \times 10^{-8}$ \\
0.9 & $3.388977 \times 10^{-6}$ & $2.093040 \times 10^{-6}$ & $1.384330 \times 10^{-6}$ & $5.057264 \times 10^{-8}$ \\
\hline
\end{tabular}

TABLE 4: Obtained absolute errors for $M=20, k=5$ and different values of $\alpha$ for Example 5.

\begin{tabular}{lcccccc}
\hline$t$ & $\alpha=1.1$ & $\alpha=1.3$ & $\alpha=1.5$ & $\alpha=1.7$ & $\alpha=1.9$ \\
\hline 0.1 & $4.3764 \times 10^{-8}$ & $1.4878 \times 10^{-7}$ & $2.0748 \times 10^{-7}$ & $1.7265 \times 10^{-7}$ & $2.5621 \times 10^{-6}$ & $5.3321 \times 10^{-10}$ \\
0.2 & $2.2918 \times 10^{-8}$ & $2.5440 \times 10^{-7}$ & $3.9721 \times 10^{-7}$ & $2.2383 \times 10^{-7}$ & $4.1274 \times 10^{-6}$ & $4.7397 \times 10^{-10}$ \\
0.3 & $5.2510 \times 10^{-9}$ & $3.3687 \times 10^{-7}$ & $5.5097 \times 10^{-7}$ & $2.3383 \times 10^{-7}$ & $5.0983 \times 10^{-6}$ & $4.1473 \times 10^{-10}$ \\
0.4 & $8.8262 \times 10^{-9}$ & $3.9515 \times 10^{-7}$ & $6.6732 \times 10^{-7}$ & $2.0325 \times 10^{-7}$ & $5.5447 \times 10^{-6}$ & $3.5549 \times 10^{-10}$ \\
0.5 & $2.0156 \times 10^{-8}$ & $4.2244 \times 10^{-7}$ & $7.2700 \times 10^{-7}$ & $1.6890 \times 10^{-7}$ & $5.5744 \times 10^{-6}$ & $2.9625 \times 10^{-10}$ \\
0.6 & $2.6409 \times 10^{-8}$ & $4.1667 \times 10^{-7}$ & $7.3063 \times 10^{-7}$ & $1.1386 \times 10^{-7}$ & $5.1809 \times 10^{-6}$ & $2.3700 \times 10^{-10}$ \\
0.7 & $2.7986 \times 10^{-8}$ & $3.7630 \times 10^{-7}$ & $6.6892 \times 10^{-7}$ & $6.2553 \times 10^{-8}$ & $4.4105 \times 10^{-6}$ & $1.7776 \times 10^{-10}$ \\
0.8 & $2.4855 \times 10^{-8}$ & $2.9259 \times 10^{-7}$ & $5.2546 \times 10^{-7}$ & $2.8409 \times 10^{-8}$ & $3.2886 \times 10^{-6}$ & $1.1851 \times 10^{-10}$ \\
0.9 & $1.5051 \times 10^{-8}$ & $1.6962 \times 10^{-6}$ & $3.0942 \times 10^{-7}$ & $4.8299 \times 10^{-9}$ & $1.8027 \times 10^{-6}$ & $5.9254 \times 10^{-11}$ \\
\hline
\end{tabular}

TABLE 5: Comparison of absolute errors between the proposed method with $M=12, k=3$, Haar wavelet method, and fourth order HPM, for different values of $\alpha$ for Example 6.

\begin{tabular}{lccc}
\hline$t$ & Current method & Haar wavelet [55] & Fourth order HPM [56] \\
\hline 0.1 & $9.0 \times 10^{-20}$ & $3.4 \times 10^{-8}$ & $5.2 \times 10^{-6}$ \\
0.2 & $2.1 \times 10^{-19}$ & $2.7 \times 10^{-8}$ & $2.7 \times 10^{-8}$ \\
0.3 & $2.1 \times 10^{-19}$ & $2.9 \times 10^{-8}$ & $1.1 \times 10^{-9}$ \\
0.4 & $2.0 \times 10^{-20}$ & $3.6 \times 10^{-8}$ & $7.9 \times 10^{-6}$ \\
0.5 & $9.0 \times 10^{-20}$ & $4.0 \times 10^{-8}$ & $3.6 \times 10^{-6}$ \\
0.6 & $5.0 \times 10^{-19}$ & $5.2 \times 10^{-8}$ & $1.4 \times 10^{-6}$ \\
0.7 & $1.1 \times 10^{-18}$ & $6.6 \times 10^{-8}$ & $5.3 \times 10^{-6}$ \\
0.8 & $1.2 \times 10^{-18}$ & $7.8 \times 10^{-8}$ & $6.5 \times 10^{-6}$ \\
0.9 & $6.2 \times 10^{-19}$ & $9.4 \times 10^{-8}$ & $4.5 \times 10^{-6}$ \\
\hline
\end{tabular}

wavelet bases $\psi(t)$ with $\psi(t / 50)$ in the discrete procedure. We applied the method described in Section 6 and got almost exact solution for $t \in[0,50]$, although other authors had solved the problem for $t \in[0,1]$ and $t \in[0,5]$. It can be seen from Figure 4 that the approximate solution and exact solution are closely overlapped for any $t \in[0,50]$.

Example 5. Consider the following boundary value problem for nonlinear fractional order differential equation:

$$
\begin{aligned}
& D^{\alpha} y(t)+e^{-2 \pi}[y(t)]^{n}=r(t), \\
& y(0)=a, \quad y(1)=b,
\end{aligned}
$$

where $1<\alpha \leq 2, a, b \in \mathbb{R}, n \in \mathbb{N}$, and $r(t)$ is a given problem. For $\alpha=1.5, n=2, a=0, b=1$, and $r(t)=$ $((15 \sqrt{ } \pi) / 8 \Gamma((7 / 2)-\alpha)) t^{(5 / 2)-\alpha}+e^{-2 \pi} t^{5}$, it can be easily verified that the exact solution is $y(t)=t^{5 / 2}$. We use the introduced scheme in Section 6 to solve this example with $M=20, k=5$. Absolute error in solution for different values of $\alpha$ is presented in Table 4 which confirm the accuracy and efficiency of the proposed method. As it is expected, the absolute error is reduced as $\alpha$ approaches an integer value. Furthermore, we plot exact and numerical solutions for $\alpha=2$ in Figure 5.

Example 6. Consider the following boundary value problem:

$$
\begin{gathered}
D^{\alpha} y(t)=D^{\beta} y(t)-g(t), \\
y(0)=0, \quad y(1)=0,
\end{gathered}
$$

where $1<\alpha \leq 2,0<\beta \leq 1$ and $g(t)=e^{t-1}+1$. The exact solution of the problem is not known generally. It can be easily verified that for $\alpha=2, \beta=1$, the exact solution is $y(t)=t\left(1-e^{t-1}\right)$. The problem (80) is solved numerically for integer order case in $[55,56]$ using Haar wavelet method and combined homotopy perturbation method, respectively. We solve the problem using $M=12, k=3$. In Table 5, the absolute errors are presented which confirm that the proposed method is more accurate. The numerical results for $\beta=1$ and different values of $\alpha$ plotted in Figure 6 show that as $\alpha$ tends to 2 , the solution of fractional differential equation approaches to that of the integer-order differential equation. 
TABLE 6: Comparison of absolute errors in $y(t)$ in [50] and the ones obtained by our method for Example 7.

\begin{tabular}{|c|c|c|c|c|c|c|c|c|}
\hline \multirow{2}{*}{$\alpha$} & \multicolumn{2}{|c|}{$t=0.1$} & \multicolumn{2}{|c|}{$t=0.3$} & \multicolumn{2}{|c|}{$t=0.7$} & \multicolumn{2}{|c|}{$t=0.9$} \\
\hline & Our result & Ref. [50] & Our result & Ref. [50] & Our result & Ref. [50] & Our result & Ref. [50] \\
\hline 0.2 & $2.1 \times 10^{-4}$ & $2.9 \times 10^{-1}$ & $7.3 \times 10^{-5}$ & $4.5 \times 10^{-1}$ & $3.1 \times 10^{-5}$ & $3.7 \times 10^{-1}$ & $2.5 \times 10^{-5}$ & $2.0 \times 10^{-1}$ \\
\hline 0.4 & $3.6 \times 10^{-4}$ & $3.9 \times 10^{-1}$ & $1.4 \times 10^{-4}$ & $5.1 \times 10^{-1}$ & $6.0 \times 10^{-5}$ & $3.3 \times 10^{-1}$ & $4.7 \times 10^{-5}$ & $2.2 \times 10^{-1}$ \\
\hline 0.6 & $3.7 \times 10^{-4}$ & $6.7 \times 10^{-3}$ & $1.7 \times 10^{-4}$ & $2.0 \times 10^{-5}$ & $7.8 \times 10^{-5}$ & $4.4 \times 10^{-3}$ & $6.0 \times 10^{-5}$ & $4.6 \times 10^{-3}$ \\
\hline 0.8 & $2.4 \times 10^{-4}$ & $1.1 \times 10^{-3}$ & $1.5 \times 10^{-4}$ & $2.1 \times 10^{-4}$ & $8.0 \times 10^{-5}$ & $8.7 \times 10^{-4}$ & $6.3 \times 10^{-5}$ & $5.8 \times 10^{-4}$ \\
\hline 1.2 & $2.5 \times 10^{-4}$ & $3.1 \times 10^{-3}$ & $1.9 \times 10^{-4}$ & $2.8 \times 10^{-3}$ & $7.1 \times 10^{-5}$ & $3.6 \times 10^{-3}$ & $2.2 \times 10^{-5}$ & $1.8 \times 10^{-3}$ \\
\hline 1.4 & $4.9 \times 10^{-5}$ & $1.0 \times 10^{-3}$ & $4.5 \times 10^{-5}$ & $7.0 \times 10^{-4}$ & $2.1 \times 10^{-5}$ & $1.1 \times 10^{-3}$ & $6.9 \times 10^{-6}$ & $2.4 \times 10^{-4}$ \\
\hline 1.6 & $1.4 \times 10^{-5}$ & $3.0 \times 10^{-4}$ & $1.6 \times 10^{-5}$ & $1.3 \times 10^{-4}$ & $8.8 \times 10^{-6}$ & $3.0 \times 10^{-4}$ & $3.0 \times 10^{-6}$ & $6.2 \times 10^{-7}$ \\
\hline 1.8 & $4.0 \times 10^{-6}$ & $6.1 \times 10^{-5}$ & $5.9 \times 10^{-6}$ & $1.4 \times 10^{-5}$ & $3.5 \times 10^{-6}$ & $5.3 \times 10^{-5}$ & $1.8 \times 10^{-6}$ & $8.8 \times 10^{-6}$ \\
\hline
\end{tabular}

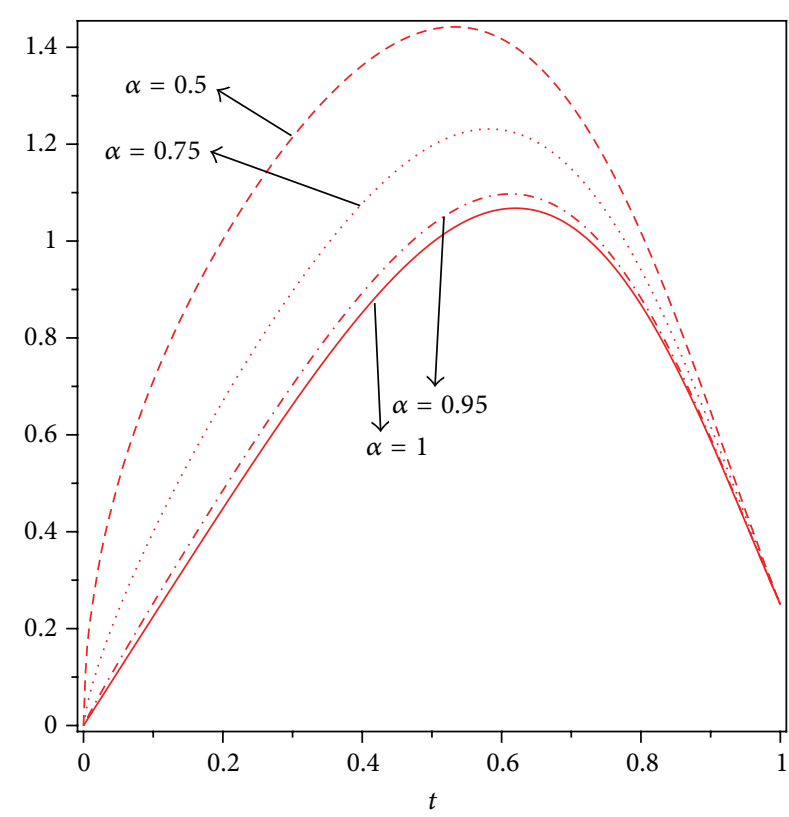

(a)

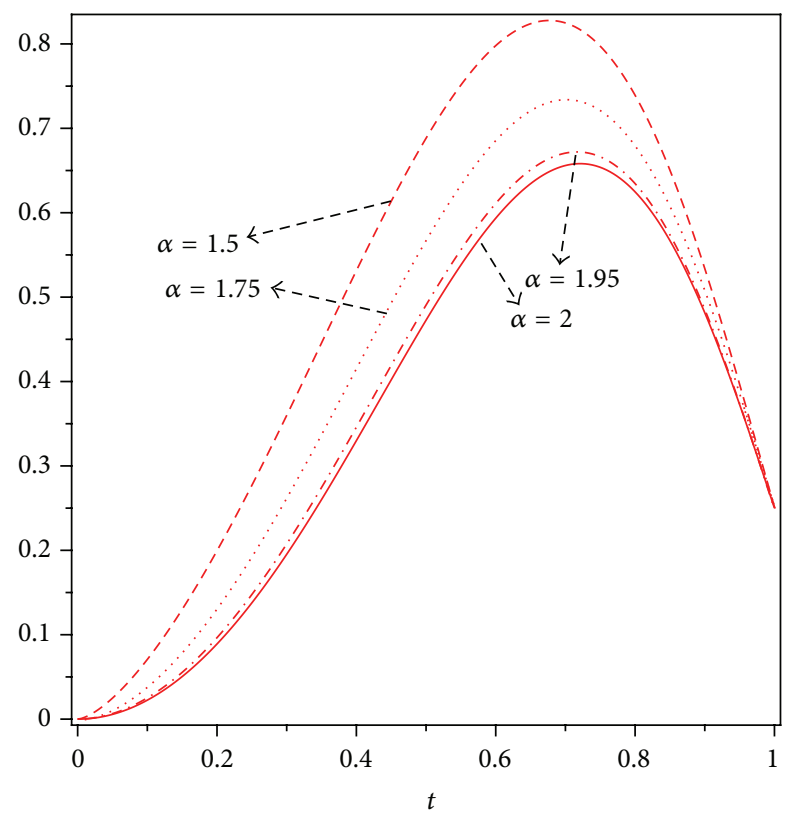

(b)

Figure 2: Comparison of $y(t)$ for $M=12, k=4$, and (a) $\alpha=0.5,0.75,0.95,1$, (b) $\alpha=1.5,1.75,1.95,2$ for Example 1.

Example 7. Consider the following linear fractional differential equation $[26,49,50,57]$ :

$$
D^{\alpha} y(t)+y(t)=0, \quad 0<\alpha \leq 2,
$$

such that

$$
y(0)=1, \quad y^{\prime}(0)=0 .
$$

The condition $y^{\prime}(0)=0$ is only for $1<\alpha \leq 2$. The exact solution of (74) and (75) is given by

$$
y(t)=E_{a}\left(-t^{\alpha}\right),
$$

where $E_{a}(z)=\sum_{k=0}^{\infty} z^{k} / \Gamma(\alpha k+1)$ is the Mittag-Leffler function of order $\alpha$. It can be easily verified that for $\alpha=1$ and $\alpha=2$, the exact solutions are $y(t)=\exp (-t)$ and $y(t)=$ $\cos (t)$, respectively.

We solved the problem using the proposed method for different values of $\alpha$.
The absolute errors for $\alpha=0.2,0.4,0.6,0.8$ (with $M=6$, $k=6$ ) and $\alpha=1.2,1.4,1.6,1.8$ (with $M=6, k=8$ ) are shown in Table 6. It can be seen that our results are more accurate than the ones reported in [50]. The numerical solutions for $\alpha=0.5,0.75,0.95$, and 1 (Figure 7(a)) and $\alpha=1.5,1.75,1.95$, and 2 (Figure 7(b)) are plotted in Figure 7. It should be noted that as $\alpha$ approaches 1 and 2 , the numerical solutions converge to the analytical solution $y(t)=\exp (-t)$ and $y(t)=\cos (t)$, respectively.

Example 8. As the last example, consider the following linear multiterm fractional boundary value problem:

$$
4(t+1) D^{2.5} y(t)+4 D^{1.5} y(t)+\frac{1}{\sqrt{t+1}} y(t)=\sqrt{t}+\sqrt{\pi}
$$

subject to

$$
y(0)=\sqrt{\pi}, \quad y^{\prime}(0)=\frac{\sqrt{\pi}}{2}, \quad y(1)=\sqrt{2 \pi} .
$$




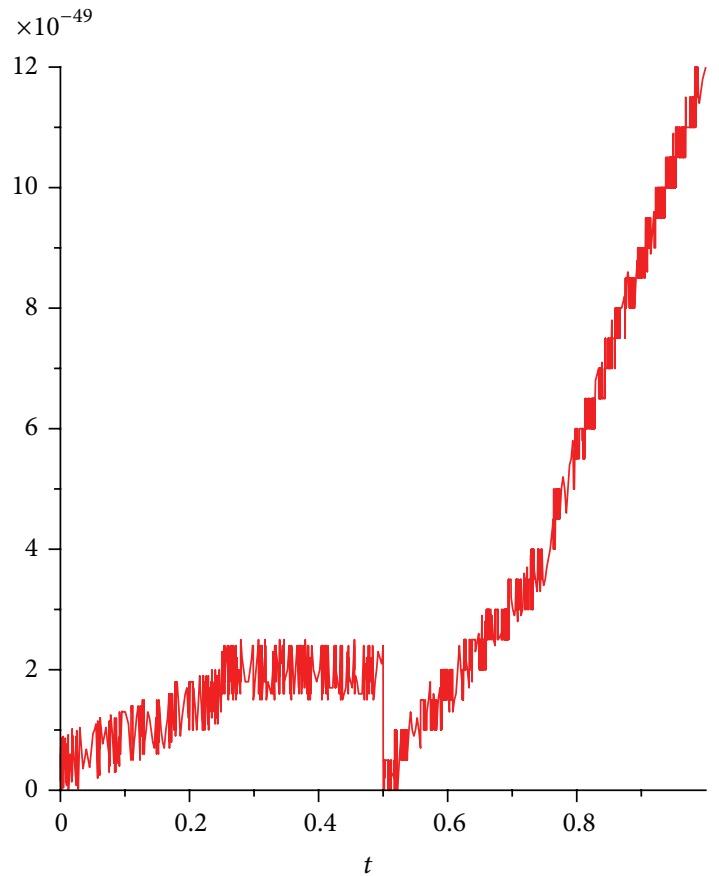

Figure 3: Absolute error for Example 2.

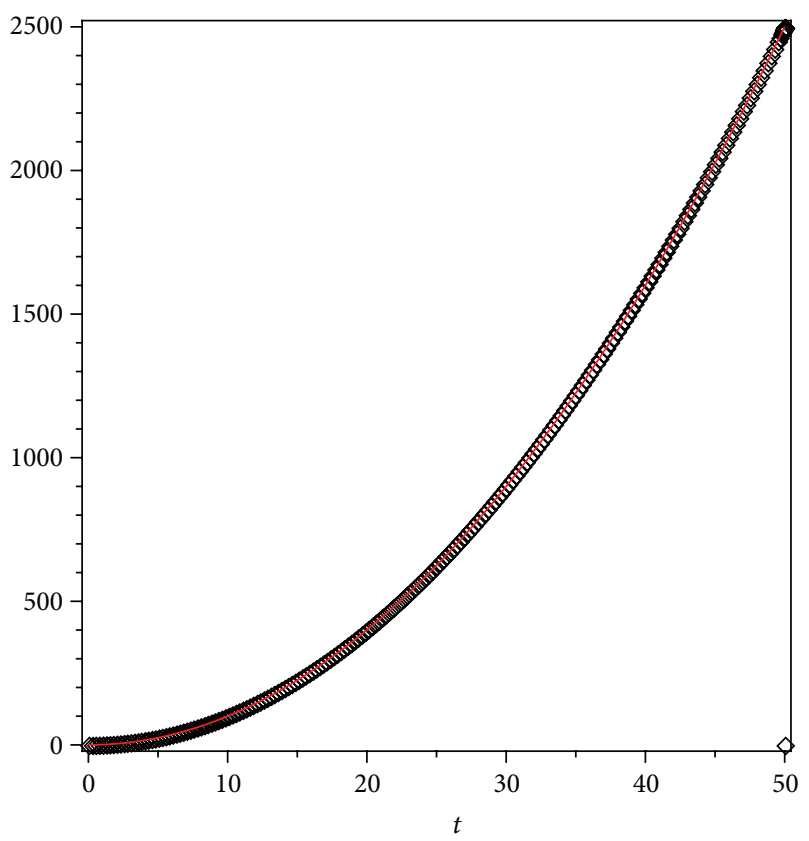

Figure 4: Approximate and exact solutions for Example 4 in the interval $[0,50]$.

The exact solution of this problem is $y(t)=\sqrt{\pi(t+1)}$. This problem was solved in [58] by operational matrix of fractional derivatives using $\mathrm{B}$-spline functions. We compare maximum absolute errors obtained by the introduced method with the ones reported in [58] in Table 7. It should be noted that the algebraic system of equations obtained by the method [58] is of order $2^{J}+1$, while the resulting one by the current method is of order $2^{k-1}(M+1)$. It can be seen from Table 7 that

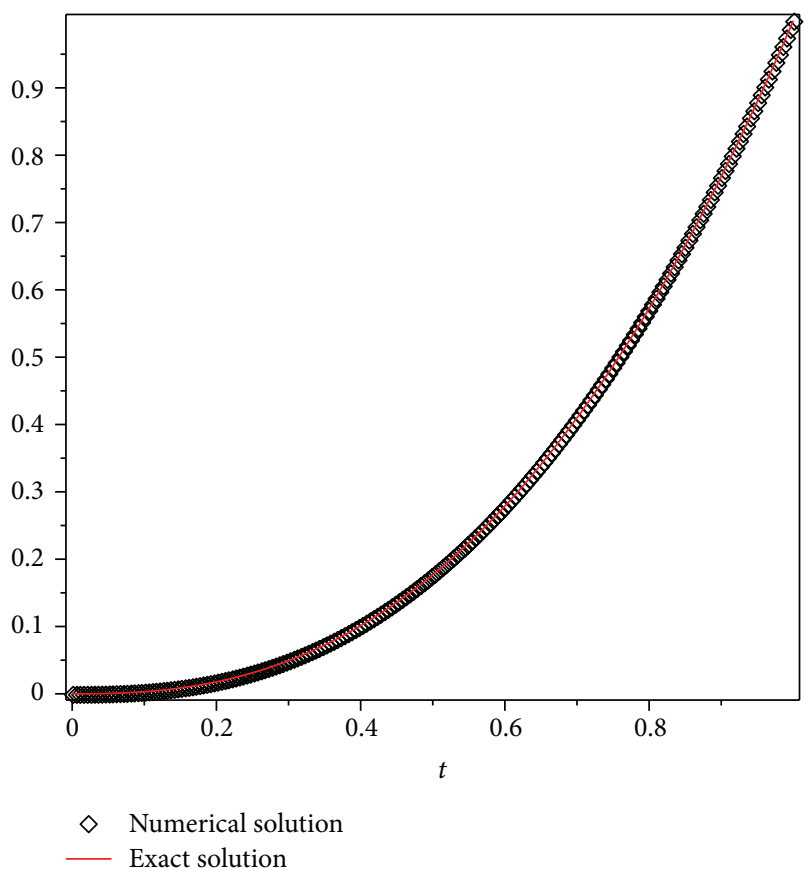

FIgURE 5: Approximate and exact solutions for $\alpha=2$ for Example 5.

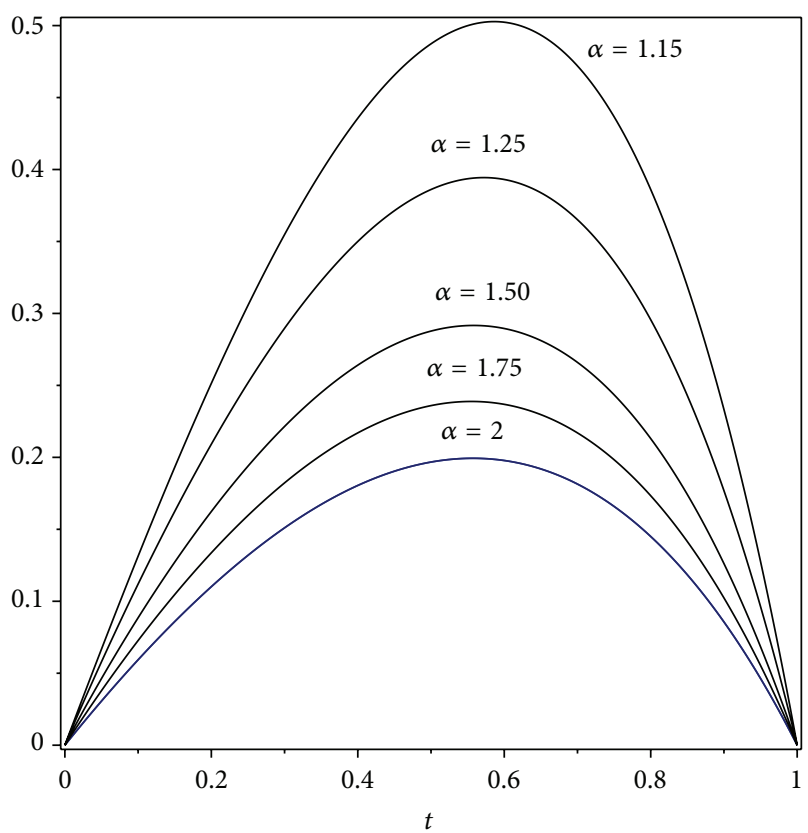

Figure 6: Chebyshev wavelet finite difference solutions for $\beta=1$ and different values of $\alpha$ for Example 6.

our results are more accurate while we need to solve a system of algebraic equations of lower order.

\section{Conclusion}

An efficient and accurate method based on hybrid of Chebyshev wavelets and finite difference methods was introduced. The fractional derivative is described in the Caputo sense. 


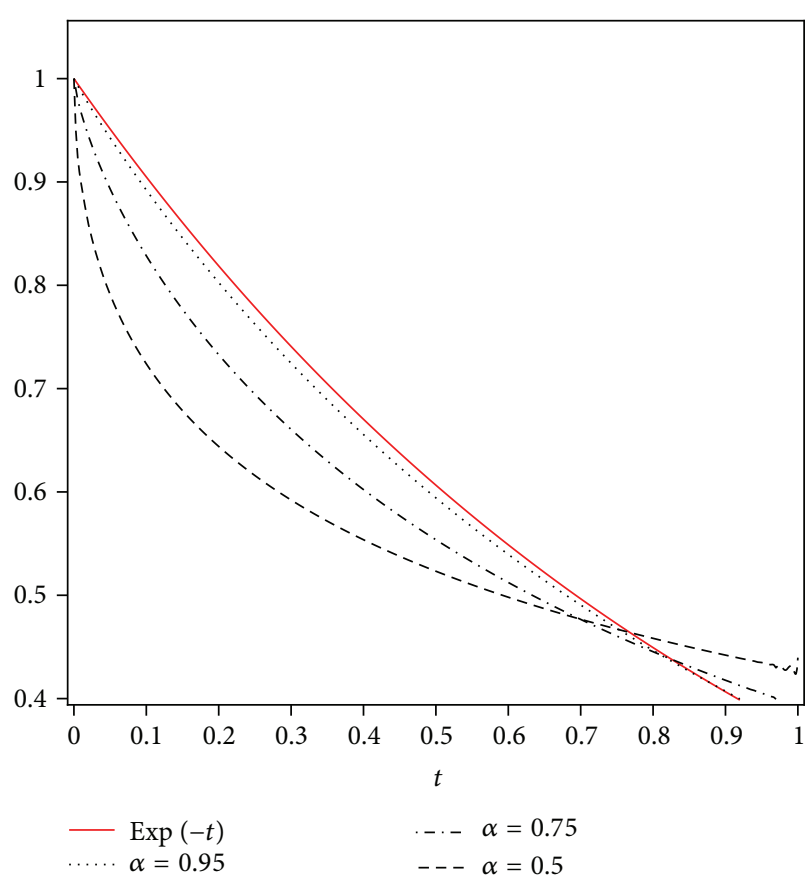

(a)

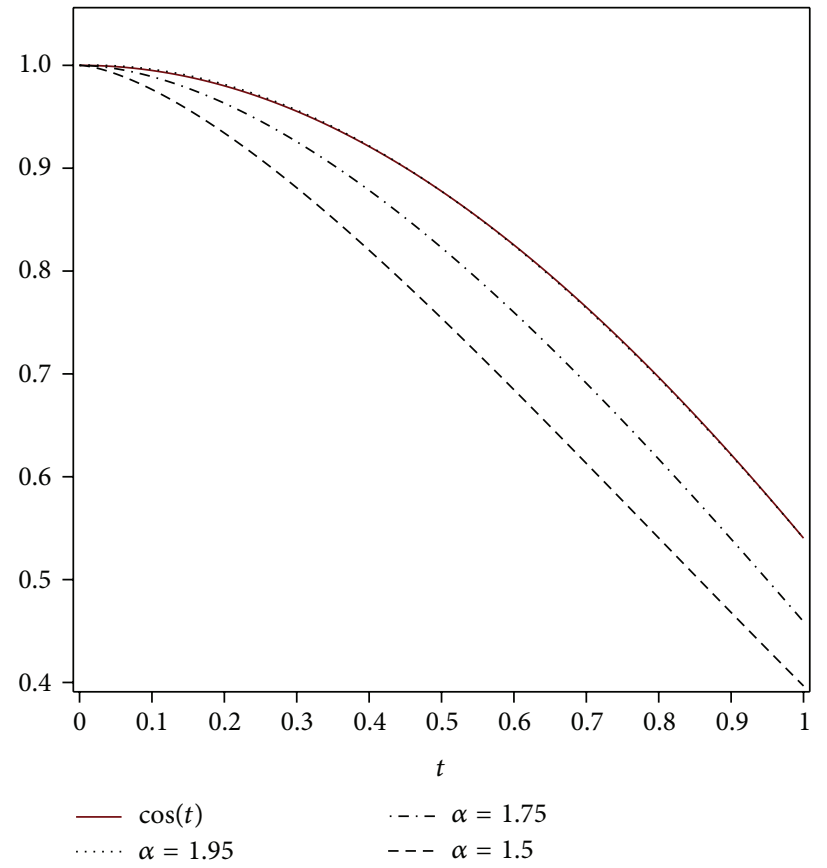

(b)

FIgURE 7: Comparison of $y(t)$ with (a) $\alpha=0.5,0.75,0.95,1$, (b) $\alpha=1.5,1.75,1.95,2$, for Example 7.

TABLE 7: Comparison of maximum absolute errors in $y(t)$ in [58] and the ones obtained by current method for Example 8.

\begin{tabular}{ccccccc}
\hline & \multicolumn{3}{c}{ Presented method } & \multicolumn{2}{c}{ Ref. [58] } \\
& $M=6$ & $M=8$ & $M=10$ & $M=12$ & $J$ & Error \\
\hline$k=2$ & $3.3 \times 10^{-7}$ & $2.8 \times 10^{-7}$ & $1.4 \times 10^{-7}$ & $8.2 \times 10^{-8}$ & 4 & $2.6 \times 10^{-4}$ \\
$k=3$ & $1.8 \times 10^{-7}$ & $8.2 \times 10^{-8}$ & $3.9 \times 10^{-8}$ & $2.2 \times 10^{-8}$ & 5 & $7.1 \times 10^{-5}$ \\
$k=4$ & $6.6 \times 10^{-8}$ & $2.4 \times 10^{-8}$ & $1.1 \times 10^{-8}$ & $6.2 \times 10^{-9}$ & 6 & $1.5 \times 10^{-5}$ \\
$k=5$ & $2.4 \times 10^{-8}$ & $8.6 \times 10^{-9}$ & $4.0 \times 10^{-9}$ & $2.2 \times 10^{-9}$ & 7 & $4.2 \times 10^{-6}$ \\
\hline
\end{tabular}

The useful properties of Chebyshev wavelets and finite difference method make it a computationally efficient method for solving the problems. The main advantage of the present method is the ability to represent smooth and especially piecewise smooth functions properly. Several examples are given to demonstrate the powerfulness of the proposed method. We note that the accuracy can be enhanced either by increasing the number of subintervals or by increasing the number of collocation points in subintervals properly. The validity and accuracy of the method were investigated for large intervals as well.

\section{Acknowledgment}

The authors gratefully acknowledge that this research was partially supported by the University Putra Malaysia under the ERGS Grant Scheme having project no. 5527068.

\section{References}

[1] P. L. Butzer and U. Westphal, An Introduction to Fractional Calculus, World Scientific, Singapore, 2000.

[2] K. S. Miller and B. Ross, An Introduction to the Fractional Calculus and Fractional Differential Equations, John Wiley \& Sons, New York, NY, USA, 1993.

[3] A. A. Kilbas, H. M. Srivastava, and J. J. Trujillo, Theory and Applications of Fractional Differential Equations, vol. 204 of North-Holland Mathematics Studies, Elsevier Science, Amsterdam, The Netherlands, 2006.

[4] J. T. Machado, V. Kiryakova, and F. Mainardi, "Recent history of fractional calculus," Communications in Nonlinear Science and Numerical Simulation, vol. 16, no. 3, pp. 1140-1153, 2011.

[5] M. Ciesielski and L. Jacek, "Numerical simulations of anomalous diffusion," Computer Methods in Mechanics, Conference Gliwice, Wisla, Poland, 2003.

[6] R. Metzler and J. Klafter, "The random walk's guide to anomalous diffusion: a fractional dynamics approach," Physics Reports, vol. 339, no. 1, pp. 1-77, 2000.

[7] I. Hashim, O. Abdulaziz, and S. Momani, "Homotopy analysis method for fractional IVPs," Communications in Nonlinear Science and Numerical Simulation, vol. 14, no. 3, pp. 674-684, 2009.

[8] O. Abdulaziz, I. Hashim, and S. Momani, "Solving systems of fractional differential equations by homotopy-perturbation method," Physics Letters A, vol. 372, no. 4, pp. 451-459, 2008.

[9] A. A. Elbeleze, A. Kılıçman, and B. M. Taib, "Applications of homotopy perturbation and variational iteration methods for fredholm integro-differential equation of fractional order," Abstract and Applied Analysis, vol. 2012, Article ID 763139, 14 pages, 2012.

[10] A. Kadem and A. K1lıçman, "The approximate solution of fractional Fredholm integrodifferential equations by variational 
iteration and homotopy perturbation methods," Abstract and Applied Analysis, vol. 2012, Article ID 486193, 10 pages, 2012.

[11] G.-C. Wu and E. W. M. Lee, "Fractional variational iteration method and its application," Physics Letters A, vol. 374, no. 25, pp. 2506-2509, 2010.

[12] Z. M. Odibat and S. Momani, "Application of variational iteration method to nonlinear differential equations of fractional order," International Journal of Nonlinear Sciences and Numerical Simulation, vol. 7, no. 1, pp. 27-34, 2006.

[13] Z. Odibat and S. Momani, "Numerical methods for nonlinear partial differential equations of fractional order," Applied Mathematical Modelling, vol. 32, no. 1, pp. 28-39, 2008.

[14] I. Podlubny, Fractional Differential Equations: An Introduction to Fractional Derivatives, Fractional Differential Equations, to Methods of Their Solution and Some of Their Applications, Academic Press, New York, NY, USA, 1999.

[15] S. B. Yuste, "Weighted average finite difference methods for fractional diffusion equations," Journal of Computational Physics, vol. 216, no. 1, pp. 264-274, 2006.

[16] Z. Odibat, "Approximations of fractional integrals and Caputo fractional derivatives," Applied Mathematics and Computation, vol. 178, no. 2, pp. 527-533, 2006.

[17] Z. M. Odibat, "Computational algorithms for computing the fractional derivatives of functions," Mathematics and Computers in Simulation, vol. 79, no. 7, pp. 2013-2020, 2009.

[18] Y. Zhang, "A finite difference method for fractional partial differential equation," Applied Mathematics and Computation, vol. 215, no. 2, pp. 524-529, 2009.

[19] V. Daftardar-Gejji and H. Jafari, "Solving a multi-order fractional differential equation using Adomian decomposition," Applied Mathematics and Computation, vol. 189, no. 1, pp. 541548, 2007.

[20] S. Momani and Z. Odibat, "Analytical solution of a timefractional Navier-Stokes equation by Adomian decomposition method," Applied Mathematics and Computation, vol. 177, no. 2, pp. 488-494, 2006.

[21] S. Momani and Z. Odibat, "Numerical approach to differential equations of fractional order," Journal of Computational and Applied Mathematics, vol. 207, no. 1, pp. 96-110, 2007.

[22] Z. Odibat and S. Momani, "Numerical methods for nonlinear partial differential equations of fractional order," Applied Mathematical Modelling, vol. 32, no. 1, pp. 28-39, 2008.

[23] C. H. Che Hussin and A. Kılıçman, "On the solutions of nonlinear higher-order boundary value problems by using differential transformation method and Adomian decomposition method," Mathematical Problems in Engineering, vol. 2011, Article ID 724927, 19 pages, 2011.

[24] Z. Odibat, S. Momani, and V. S. Erturk, "Generalized differential transform method: application to differential equations of fractional order," Applied Mathematics and Computation, vol. 197, no. 2, pp. 467-477, 2008.

[25] C. H. C. Hussin and A. K1lıçman, "On the solution of fractional order nonlinear boundary value problems by using differential transformation method," European Journal of Pure and Applied Mathematics, vol. 4, no. 2, pp. 174-185, 2011.

[26] K. Diethelm, N. J. Ford, and A. D. Freed, "A predictor-corrector approach for the numerical solution of fractional differential equations," Nonlinear Dynamics, vol. 29, no. 1-4, pp. 3-22, 2002.

[27] Ch. Lubich, "Fractional linear multistep methods for AbelVolterra integral equations of the second kind," Mathematics of Computation, vol. 45, no. 172, pp. 463-469, 1985.
[28] K. Diethelm and G. Walz, "Numerical solution of fractional order differential equations by extrapolation," Numerical Algorithms, vol. 16, no. 3-4, pp. 231-253, 1997.

[29] A. Kadem and A. Kılıçman, "Note on transport equation and fractional Sumudu transform," Computers \& Mathematics with Applications, vol. 62, no. 8, pp. 2995-3003, 2011.

[30] Y. Li and N. Sun, "Numerical solution of fractional differential equations using the generalized block pulse operational matrix," Computers \& Mathematics with Applications, vol. 62, no. 3, pp. 1046-1054, 2011.

[31] A. Kilıçman and Z. A. A. Al Zhour, "Kronecker operational matrices for fractional calculus and some applications," Applied Mathematics and Computation, vol. 187, no. 1, pp. 250-265, 2007.

[32] M. Misiti, Y. Misiti, G. Oppenheim, and J.-M. Poggi, Wavelets Toolbox Users Guide, The MathWorks, 2000, Wavelet Toolbox, for use with Matlab.

[33] Y. Li, "Solving a nonlinear fractional differential equation using Chebyshev wavelets," Communications in Nonlinear Science and Numerical Simulation, vol. 15, no. 9, pp. 2284-2292, 2010.

[34] H. Jafari, S. A. Yousefi, M. A. Firoozjaee, S. Momani, and C. M. Khalique, "Application of Legendre wavelets for solving fractional differential equations," Computers \& Mathematics with Applications, vol. 62, no. 3, pp. 1038-1045, 2011.

[35] Y. L. Li and W. W. Zhao, "Haar wavelet operational matrix of fractional order integration and its applications in solving the fractional order differential equations," Applied Mathematics and Computation, vol. 216, no. 8, pp. 2276-2285, 2010.

[36] H. Saeedi, M. Mohseni Moghadam, N. Mollahasani, and G. N. Chuev, "A CAS wavelet method for solving nonlinear Fredholm integro-differential equations of fractional order," Communications in Nonlinear Science and Numerical Simulation, vol. 16, no. 3, pp. 1154-1163, 2011.

[37] H. Saeedi and M. M. Moghadam, "Numerical solution of nonlinear Volterra integro-differential equations of arbitrary order by CAS wavelets," Communications in Nonlinear Science and Numerical Simulation, vol. 16, no. 3, pp. 1216-1226, 2011.

[38] S. G. Samko, A. A. Kilbas, and O. I. Marichev, Fractional Integrals and Derivatives: Theory and Applications, Gordon and Breach, 1993.

[39] R. Hilfer, Applications of Fractional Calculus in Physics, World Scientific, River Edge, NJ, USA, 2000.

[40] J. A. Tenreiro Machado, "Fractional derivatives: probability interpretation and frequency response of rational approximations," Communications in Nonlinear Science and Numerical Simulation, vol. 14, no. 9-10, pp. 3492-3497, 2009.

[41] J. C. Mason and D. C. Handscomb, Chebyshev Polynomials, Chapman \& Hall, 2003.

[42] I. Daubechies, Ten Lectures on Wavelets, vol. 61 of CBMS-NSF Regional Conference Series in Applied Mathematics, Society for Industrial and Applied Mathematics (SIAM), Philadelphia, Pa, USA, 1992.

[43] Q. B. Fan, Wavelet Analysis, Wuhan University Press, Wuhan, China, 2008.

[44] H. Derili and S. Sohrabi, "Numerical solution of singular integral equations using orthogonal functions," Mathematical Sciences, vol. 2, no. 3, pp. 261-272, 2008.

[45] C. W. Clenshaw and A. R. Curtis, "A method for numerical integration on an automatic computer," Numerische Mathematik, vol. 2, pp. 197-205, 1960.

[46] E. M. E. Elbarbary and M. El-Kady, "Chebyshev finite difference approximation for the boundary value problems," Applied 
Mathematics and Computation, vol. 139, no. 2-3, pp. 513-523, 2003.

[47] H. Adibi and P. Assari, "Chebyshev wavelet method for numerical solution of Fredholm integral equations of the first kind," Mathematical Problems in Engineering, vol. 2010, Article ID 138408, 17 pages, 2010.

[48] P. J. Davis and P. Rabinowitz, Method of Numerical Integration, Academic Press, London, UK, 2nd edition, 1984.

[49] P. Kumar and O. P. Agrawal, "An approximate method for numerical solution of fractional differential equations," Signal Processing, vol. 86, no. 10, pp. 2602-2610, 2006.

[50] A. Saadatmandi and M. Dehghan, "A new operational matrix for solving fractional-order differential equations," Computers \& Mathematics with Applications, vol. 59, no. 3, pp. 1326-1336, 2010.

[51] Y. Wang and Q. Fan, "The second kind Chebyshev wavelet method for solving fractional differential equations," Applied Mathematics and Computation, vol. 218, no. 17, pp. 8592-8601, 2012.

[52] A. E. M. El-Mesiry, A. M. A. El-Sayed, and H. A. A. ElSaka, "Numerical methods for multi-term fractional (arbitrary) orders differential equations," Applied Mathematics and Computation, vol. 160, no. 3, pp. 683-699, 2005.

[53] Q. M. Al-Mdallal, M. I. Syam, and M. N. Anwar, "A collocationshooting method for solving fractional boundary value problems," Communications in Nonlinear Science and Numerical Simulation, vol. 15, no. 12, pp. 3814-3822, 2010.

[54] F. Mohammadi, M. M. Hosseini, and S. T. Mohyud-Din, "A new operational matrix for legendre wavelets and its applications for solving fractional order boundary values problems," International Journal of Physical Sciences, vol. 6, no. 32, pp. 7371-7378, 2011.

[55] M. ur Rehman and R. A. Khan, "A numerical method for solving boundary value problems for fractional differential equations," Applied Mathematical Modelling, vol. 36, no. 3, pp. 894-907, 2012.

[56] Y.-G. Wang, H.-F. Song, and D. Li, "Solving two-point boundary value problems using combined homotopy perturbation method and Green's function method," Applied Mathematics and Computation, vol. 212, no. 2, pp. 366-376, 2009.

[57] M. ur Rehman and R. Ali Khan, "The Legendre wavelet method for solving fractional differential equations," Communications in Nonlinear Science and Numerical Simulation, vol. 16, no. 11, pp. 4163-4173, 2011.

[58] M. Lakestani, M. Dehghan, and S. Irandoust-pakchin, "The construction of operational matrix of fractional derivatives using B-spline functions," Communications in Nonlinear Science and Numerical Simulation, vol. 17, no. 3, pp. 1149-1162, 2012. 


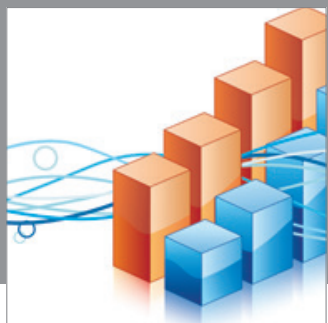

Advances in

Operations Research

mansans

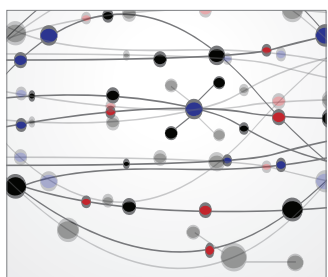

The Scientific World Journal
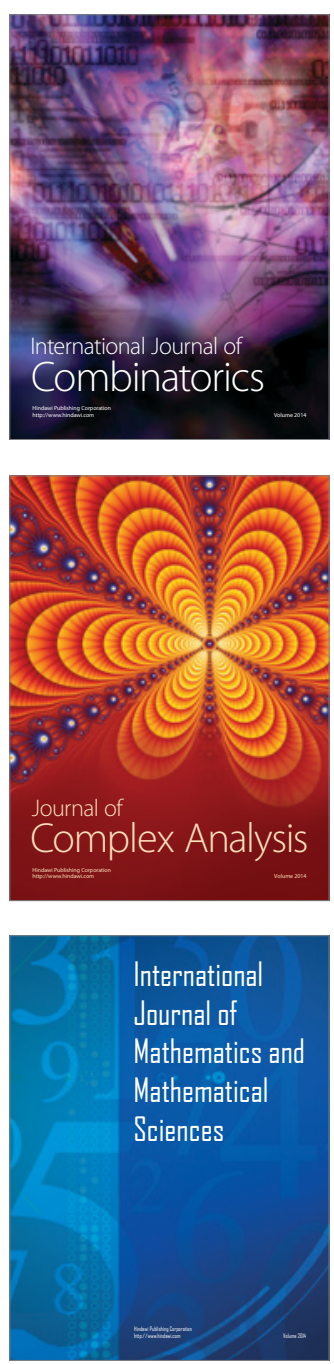
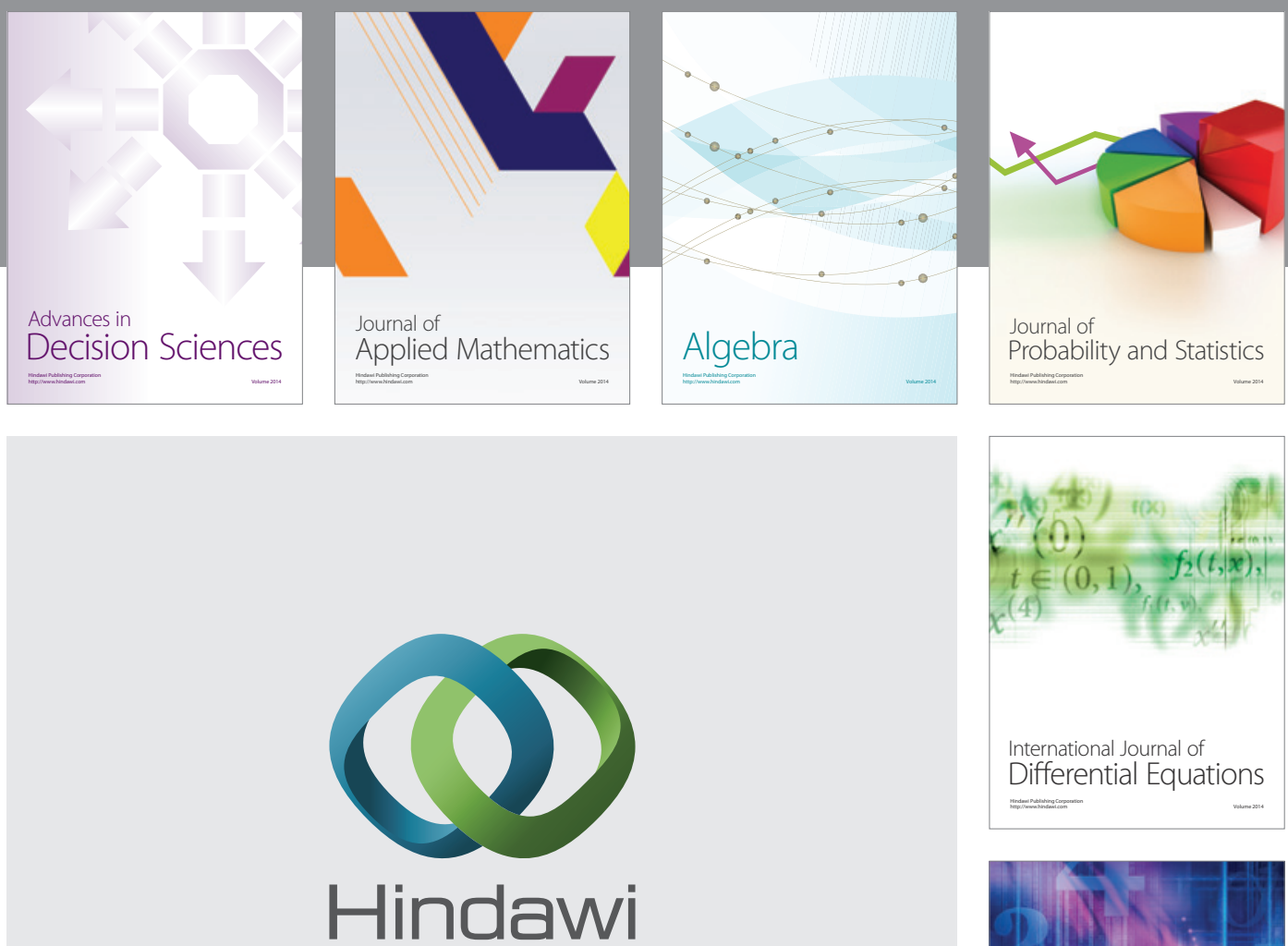

Submit your manuscripts at http://www.hindawi.com
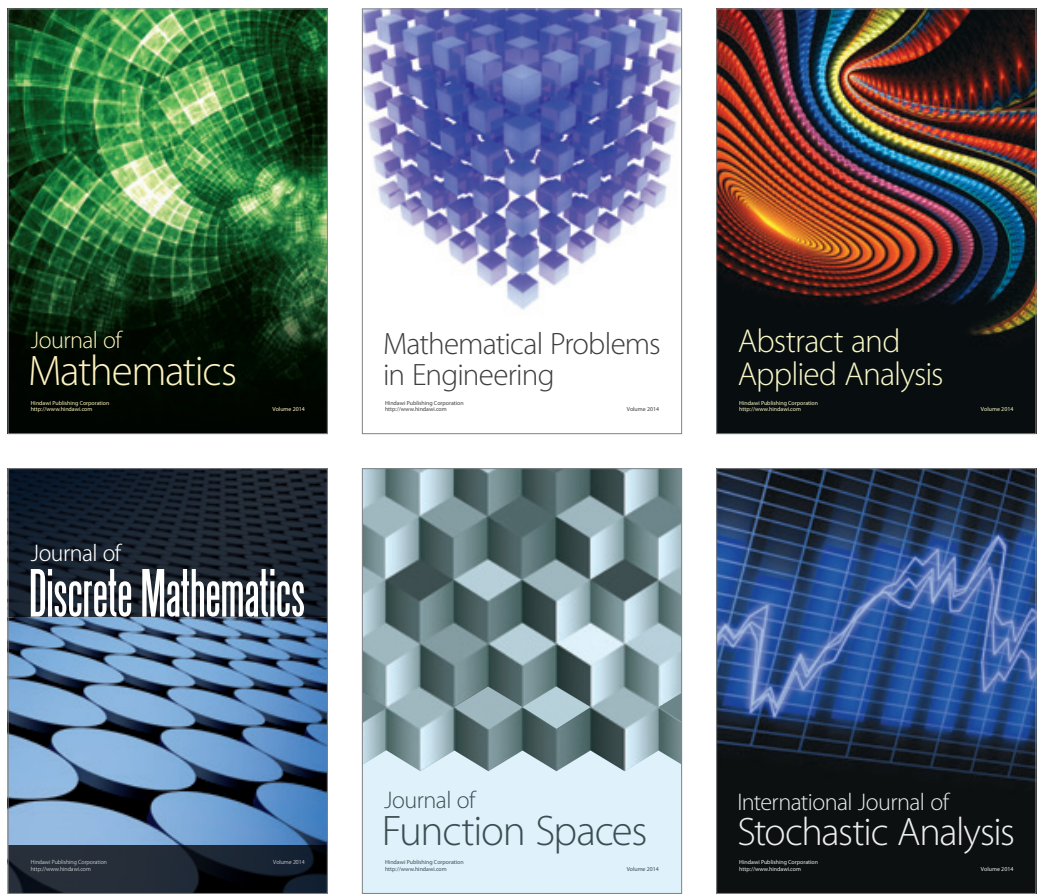

Journal of

Function Spaces

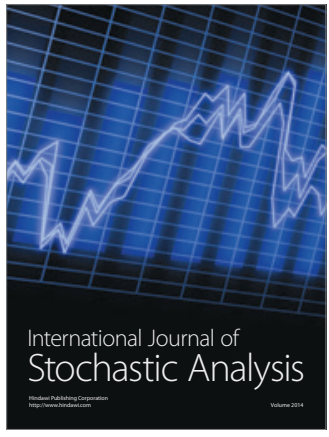

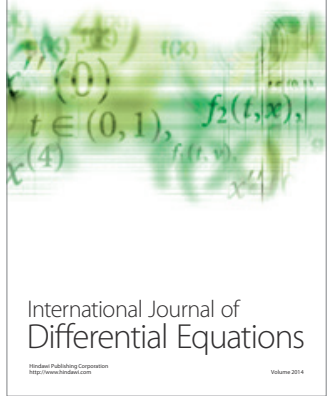
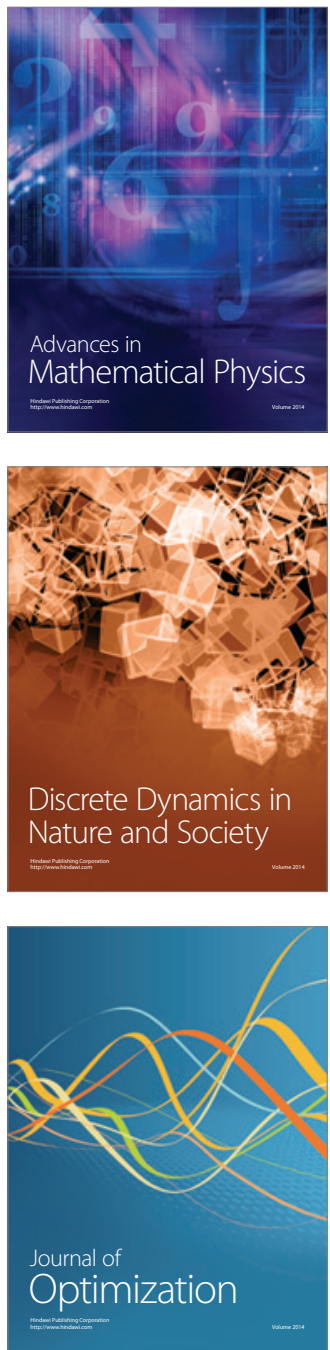